\title{
An Update on 2,5-Diketopiperazines from Marine Organisms
}

\section{Ri-Ming Huang ${ }^{1,2}$, Xiang-Xi Yi ${ }^{3}$, Yuying Zhou ${ }^{4}$, Xiangdong $\mathrm{Su}^{2}$, Yan Peng ${ }^{5}$ and Cheng-Hai Gao ${ }^{6, *}$}

1 Key Laboratory of Plant Resources Conservation and Sustainable Utilization, South China Botanical Garden, Chinese Academy of Sciences, Guangzhou 510650, China; E-Mail: huangriming@scib.ac.cn

2 Department of Pharmacy and Pharmacology, University of Bath, Bath BA2 7AY, UK;

E-Mail: prsxs@bath.ac.uk

3 School of Pharmaceutical Sciences, Guangxi University of Chinese Medicine, Nanning 530001, China; E-Mail: xiangxiyi81@aliyun.com

4 Department of Cell Biology, Jinan University, Guangzhou 510632, China; E-Mail: chenlu0268@163.com

5 Life Science \& Technology School, Lingnan Normal University, Zhanjiang 52048, China; E-Mail: py00_2006@126.com

6 Key Laboratory of Marine Environmental Science, Guangxi Academy of Sciences, Nanning 530007, China

* Author to whom correspondence should be addressed; E-Mail: gaochenghai@ gxas.cn; Tel./Fax: +86-771-2503975.

External Editor: Jordan K. Zjawiony

Received: 14 September 2014; in revised form: 5 December 2014 / Accepted: 9 December 2014 / Published: 19 December 2014

\begin{abstract}
Diketopiperazines (2,5-DKPs) are an important category of structurally diverse cyclic dipeptides with prominent biological properties. These 2,5-DKPs have been obtained from a variety of natural resources, including marine organisms. Because of the increasing numbers and biological importance of these compounds, this review covers 90 marine originated 2,5-DKPs that were reported from 2009 to the first half-year of 2014. The review will focus on the structure characterizations, biological properties and proposed biosynthetic processes of these compounds.
\end{abstract}

Keywords: 2,5-diketopiperazine; marine organism; biosynthetic processes; biological activity 


\section{Introduction}

2,5-Diketopiperazines (2,5-DKPs) are important cyclodipeptides derived from the "head to tail" cyclization of two $\alpha$-amino acids. These molecules with the double lactam core structure of 2,5-DKPs, have previously been isolated from a variety of natural resources, including marine organisms. These small, conformationally rigid, chiral templates have multiple sites in 2,5-DKPs for the structural elaboration of diverse functional groups with defined stereochemistry. These characteristics not only enable them to show a broad range of biological activities [1], but also allow the development of the drug-like physicochemical properties. The structures, reactions, medicinal chemical properties and potential therapeutic applications of 2,5-DKPs, particularly that with the interesting biological activities have previously been reviewed [2,3]. However, 2,5-DKPs belong to a relatively unexplored category of the bioactive cyclic peptides that may hold a great promise for the potential medicinal use in the future. Our previous review [1] focused on the marine-derived 2,5-DKPs, covering their structures, names, biological studies and proposed biosynthetic process. This review aims to summarize 90 marine organisms-derived 2,5-DKPs published from 2009 to the first half year of 2014. This update is taxonomically presented based on the origin of the isolation of these 2,5-DKPs.

\section{Marine Microorganisms}

\subsection{Actinomycetes}

Eight 2,5-DKPs (1-8) have been obtained from actinomycetes (Figure 1 and Table 1). Naseseazines A (1) and B (2), with a new dimeric structural backbone, were isolated from the culture of Streptomyces sp. (sediment, Fiji), and a plausible biosynthetic route to the naseseazines and other related dimeric DKPs was also proposed (Scheme 1) [4]. The DKP derivative, nocazine C (3) was isolated from the actinomycete Nocardiopsis dassonvillei (sediment, Yellow River estuary, Dongying, China) [5]. The structure of nocazine C (3) had previously been reported [6], however, no experimental data were released to support the proposal structure [5]. In addition, five more DKP derivatives, (3Z,6E)-1- $N$-methyl-3-benzylidene-6-(2S-methyl-3-hydroxypropylidene)piperazine-2,5-dione (4), (3Z,6E)-1-N-methyl-3-benzylidene-6-(2R-methyl-3-hydroxypropylidene)piperazine-2,5-dione (5), (3Z,6Z)-3-(4-hydroxybenzylidene)-6-isobutylidenepiperazine-2,5-dione (6), (3Z,6Z)-3-((1H-imidazol5-yl)-methylene)-6-isobutylidenepiperazine-2,5-dione (7) and (3Z,6S)-3-benzylidene-6-(2S-but-2-yl) piperazine-2,5-dione (8) were isolated from the actinomycete Streptomyces sp. FXJ7.328 (sediment, Huanghai beach, China) [7].

\subsection{Bacteria}

Six 2,5-DKPs (9-14) have been obtained from marine-derived bacteria (Figure 2 and Table 2). Both bacillusamides A (9) and B (10) were isolated from the Bacillus sp. (sea urchin Anthocidaris crassispina, Nagasaki Shitsu coast, Japan). It was reported that bacillusamide A (9) exhibited a modest inhibitory property against Aspergillus niger [8]. The prenylated DKPs, norcardioazines A (11) and B (12) were obtained from the Nocardiopsis sp. (sediment, South Molle, Is., Brisbane, Australia), of which norcardioazine A (11) was found to be a noncytotoxic MDR reversing agent [9]. Furthermore, two 
DKP derivatives, staphyloamides A (13) and B (14) were isolated from the culture broth of Staphylococcus sp. (algae Corallina officinalis Lineaus, Nagasaki Shitsu coast of Japan) [10].

Figure 1. Structures of 2,5-DKPs from marine-derived actinomycetes.

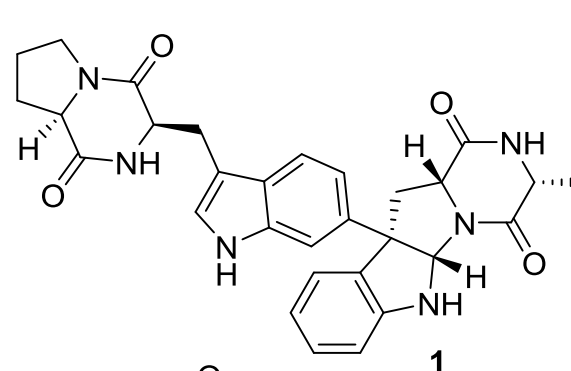

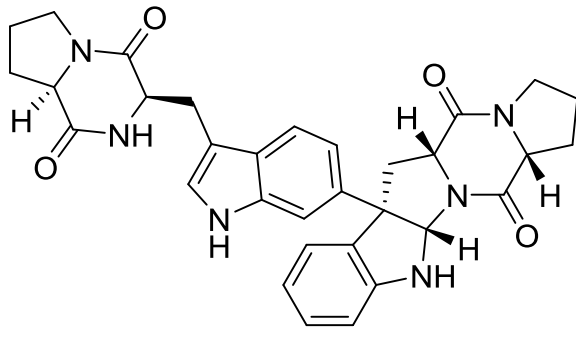

2<smiles>COc1ccc(/C=C2/C(=O)NC(Cc3ccccc3)C(=O)N2C)cc1</smiles>

3<smiles>CC(/C=c1\c(=O)[nH]/c(=C\c2ccccc2)c(=O)n1C)CO</smiles><smiles>C[C@@H](/C=c1/c(=O)[nH]/c(=C\c2ccccc2)c(=O)n1C)CO</smiles><smiles>CC(C)/C=c1\[nH]c(=O)/c(=C/c2ccc(O)cc2)[nH]c1=O</smiles><smiles>CC(C)/C=c1\[nH]c(=O)/c(=C/c2cnc[nH]2)[nH]c1=O</smiles>

7<smiles>CCC[C@H](C)C1NC(=O)/C(=C/c2ccccc2)NC1=O</smiles>

8

Table 1. 2,5-DKPs from marine-derived actinomycetes.

\begin{tabular}{|c|c|c|c|c|}
\hline Number & Name & Bioactivity & Source & Reference(s) \\
\hline 1 & Naseseazine A & - & Streptomyces sp. & [4] \\
\hline 2 & Naseseazine B & - & Streptomyces sp. & [4] \\
\hline 3 & Nocazine $\mathrm{C}$ & - & $\begin{array}{c}\text { Nocardiopsis } \\
\text { dassonvillei }\end{array}$ & {$[5,6]$} \\
\hline 4 & $\begin{array}{l}\text { (3Z,6E)-1- } N \text {-methyl-3-benzylidene-6-( } 2 S \text { - } \\
\text { methyl-3-hydroxypropylidene)piperazine- } \\
\text { 2,5-dione }\end{array}$ & - & Streptomyces sp. & [7] \\
\hline 5 & $\begin{array}{l}(3 Z, 6 E)-1-N \text {-methyl-3-benzylidene-6-(2R- } \\
\text { methyl-3-hydroxypropylidene)piperazine- } \\
\text { 2,5-dione }\end{array}$ & - & Streptomyces sp. & [7] \\
\hline 6 & $\begin{array}{l}\text { (3Z,6Z)-3-(4-hydroxybenzylidene)-6- } \\
\text { isobutylidenepiperazine-2,5-dione }\end{array}$ & $\begin{array}{l}\text { Modest antivirus } \\
\text { activity against } \\
\text { influenza A } \\
\text { (H1N1) virus }\end{array}$ & Streptomyces sp. & [7] \\
\hline 7 & $\begin{array}{l}\text { (3Z,6Z)-3-((1H-imidazol-5-yl)-methylene)- } \\
\text { 6-isobutylidenepiperazine-2,5-dione (7) }\end{array}$ & - & Streptomyces sp. & [7] \\
\hline 8 & $\begin{array}{l}\text { (3Z,6S)-3-benzylidene-6-( } 2 S \text {-but-2- } \\
\text { yl)piperazine-2,5-dione }\end{array}$ & - & Streptomyces sp. & [7] \\
\hline
\end{tabular}


Scheme 1. A plausible biosynthetic route to naseseazines A (1) and (2) and other related DKPs [4].

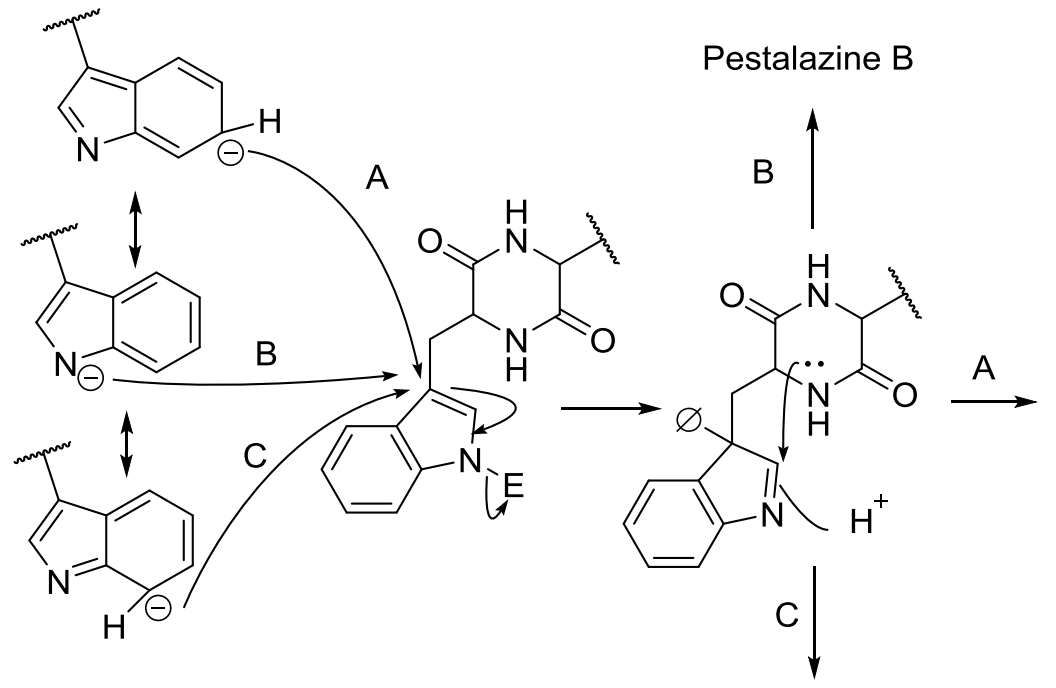

Asperazine

Pestalazine A
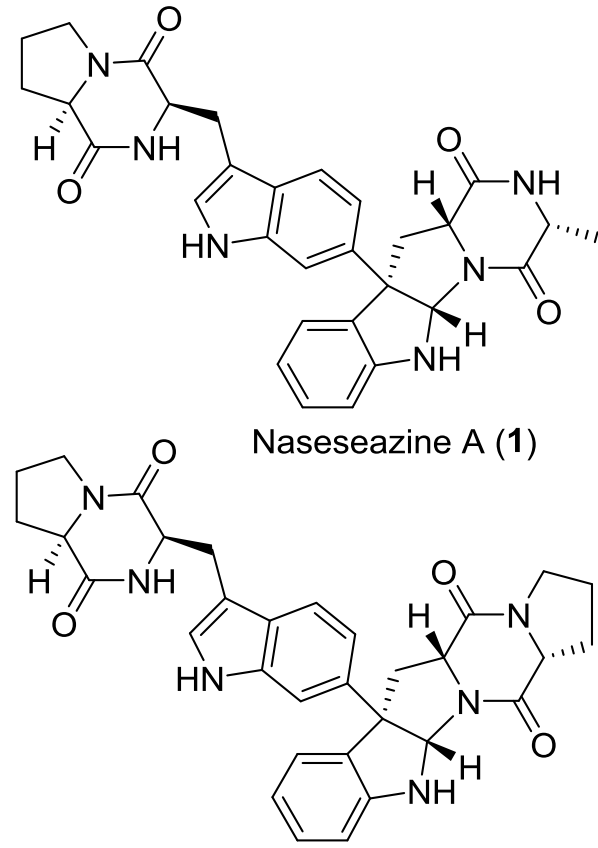

Naseseazine B (2)

Figure 2. Structures of 2,5-DKPs from marine-derived bacteria.

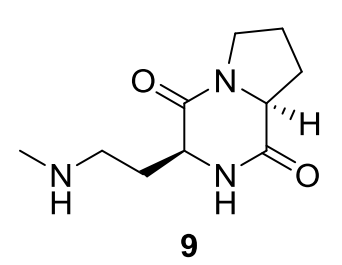

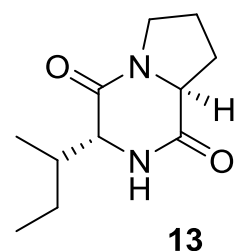<smiles>CC(C)[C@H]1NC(=O)C2(O)CCCN2C1=O</smiles>

10<smiles>CC(C)[C@H]1NC(=O)[C@]2(O)CCCN2C1=O</smiles>

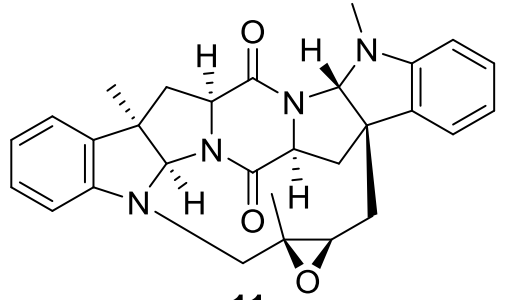

11

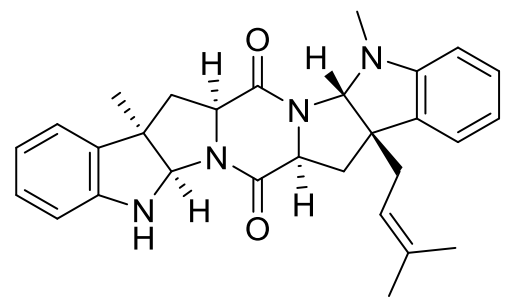

12

Table 2. 2,5-DKPs from marine-derived bacteria.

\begin{tabular}{ccccc}
\hline Number & Name & Bioactivity & Source & Reference \\
\hline $\mathbf{9}$ & Bacillusamide A & Weak inhibition activity & Bacillus sp. & {$[8]$} \\
$\mathbf{1 0}$ & Bacillusamide B & against A. niger & Bacillus sp. & {$[8]$} \\
$\mathbf{1 1}$ & Norcardioazine A & Inhibition of P-Glycoprotein & Nocardiopsis sp. & {$[9]$} \\
$\mathbf{1 2}$ & Norcardioazine B & - & Nocardiopsis sp. & {$[9]$} \\
$\mathbf{1 3}$ & Staphyloamide A & - & Staphylococcus sp. & {$[10]$} \\
$\mathbf{1 4}$ & Staphyloamide B & - & Staphylococcus sp. & {$[10]$} \\
\hline
\end{tabular}




\subsection{Fungi}

In the past five years, marine-derived fungi have been shown to be the rich sources of 2,5-DKP derivatives. Seventy-one 2,5-DKPs (15-85) have been obtained from the marine fungi (Figures 3-10 and Tables 3-10).

Figure 3. Structures of 2,5-DKPs from marine-derived fungi of sediment origin.
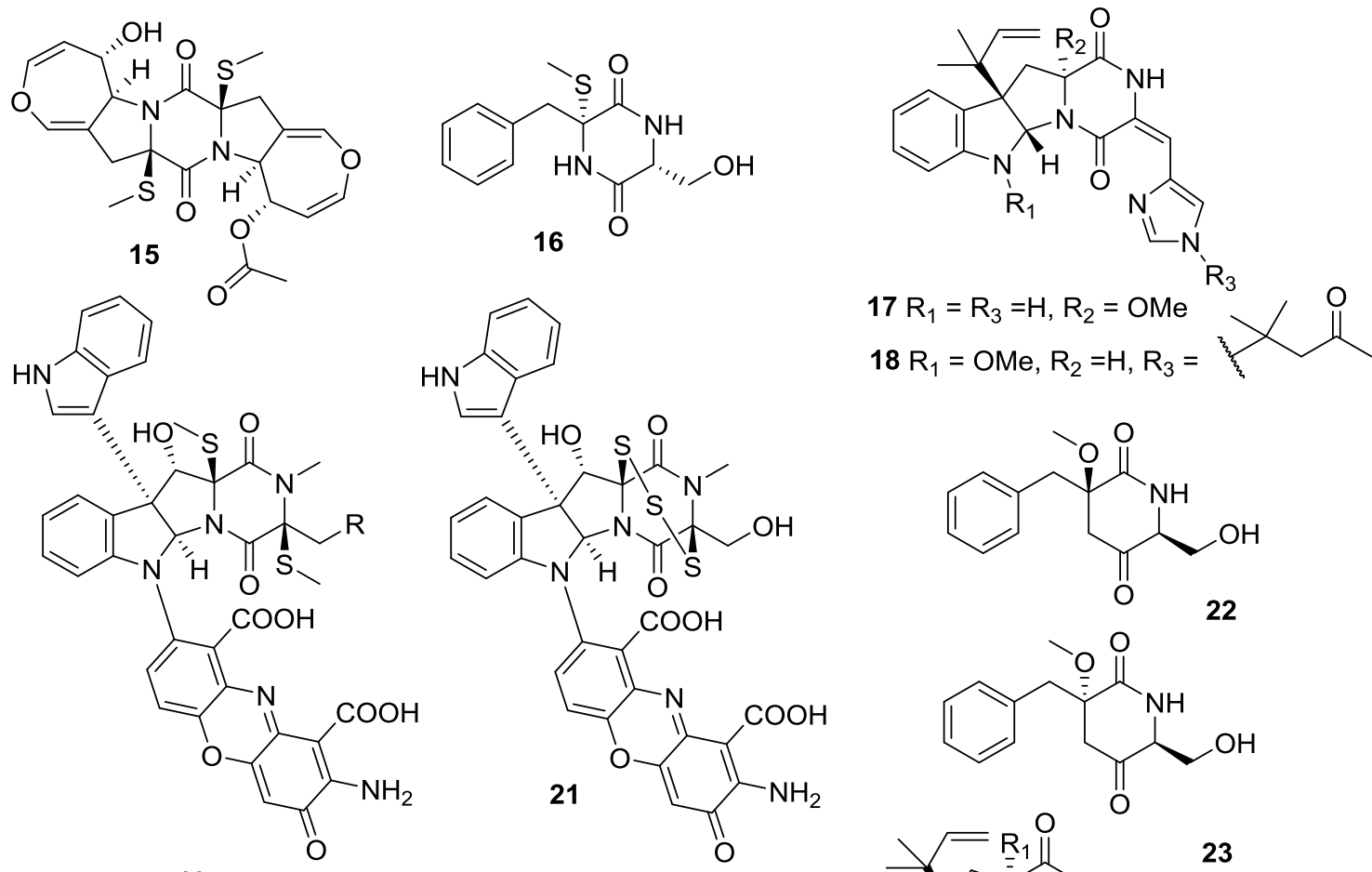<smiles>O=C1NC(O)(CO)C(=O)CC1=Cc1ccccc1</smiles>
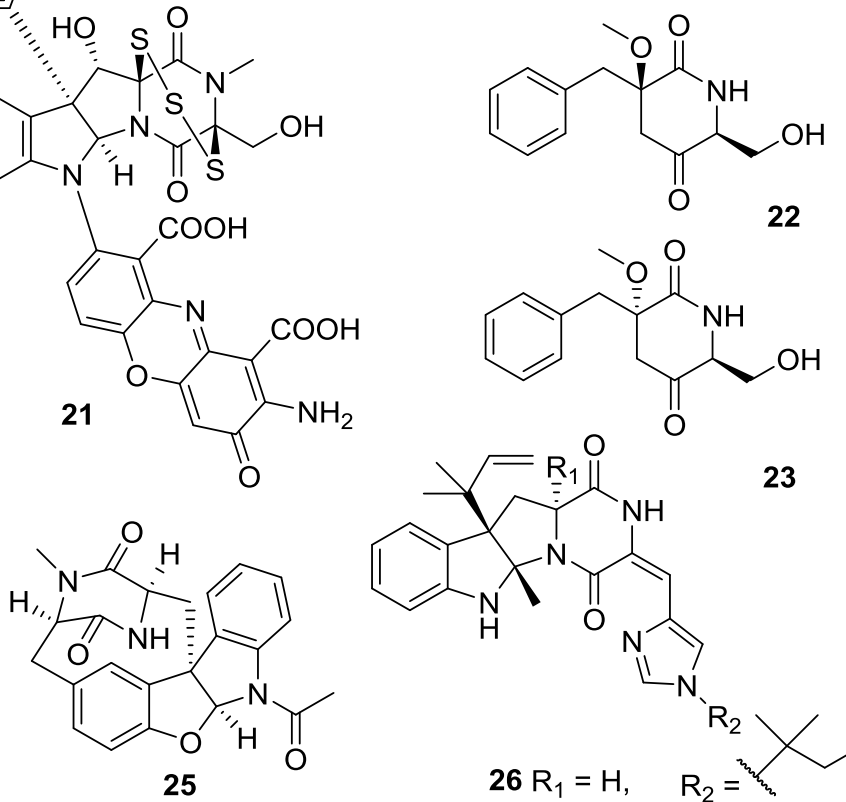<smiles>[R]CC(C)(C)CCC(=O)C1CC(=O)C(CO)NC1=O</smiles>

24

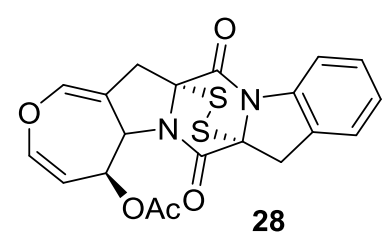

28<smiles>[R6][C@]12CC[C@H]3c4ccccc4N(C(C)=O)[C@H]3N1C(=O)[C@H](CCC)NC2=O</smiles>

$29 \mathrm{R}=\mathrm{H}$

$30 \mathrm{R}=\mathrm{Ac}$<smiles>C=CC(C)(C)c1[nH]c2ccccc2c1C=c1[nH]c(=O)c2n(c1=O)CCC=2C1=c2c(=O)[nH]/c(=C\c3c(C(C)(C)C=C)[nH]c4ccccc34)c(=O)n2CC1</smiles>

$32 n=3$

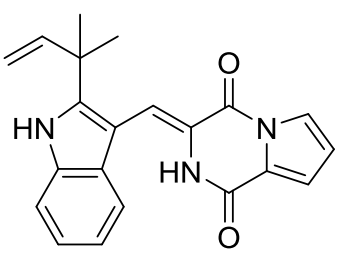


Figure 3. Cont.

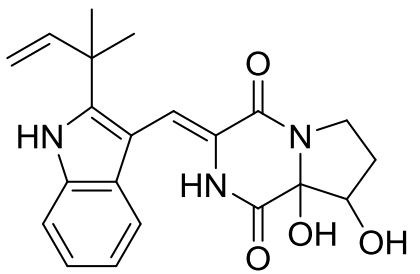

35

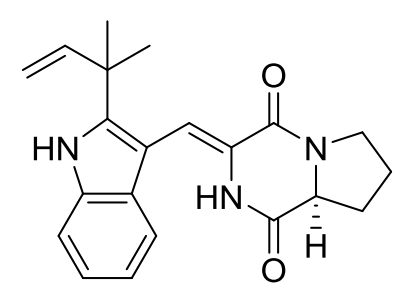

36

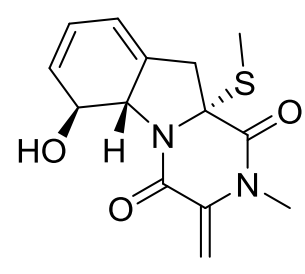

37

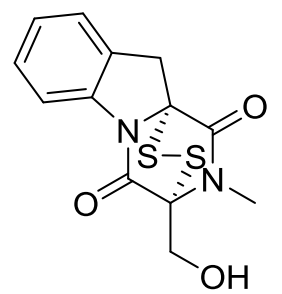

38

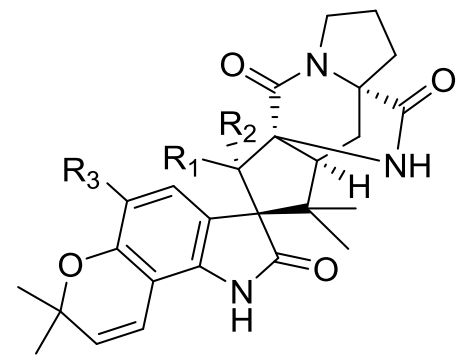<smiles>COc1ccc(/C=C2\NC(=O)[C@H](Cc3ccccc3)NC2=O)cc1</smiles><smiles>COc1ccc(/C=C2\NC(=O)[C@H](Cc3ccccc3)NC2=O)cc1</smiles>

$39 \mathrm{R}_{1}=\mathrm{H}, \mathrm{R}_{2}=\mathrm{OH}, \mathrm{R}_{3}=\mathrm{Cl}$

$40 \mathrm{R}_{1}=\mathrm{OH}, \mathrm{R}_{2}=\mathrm{H}, \mathrm{R}_{3}=\mathrm{H}$

Figure 4. Structures of 2,5-DKPs from marine-derived fungi of algae origin.<smiles>C=CC(C)(C)c1[nH]c2cc3c(cc2c1C[C@@H]1NC(=O)C2=CCCN2C1=O)C=CC(C)(C)O3</smiles>

43

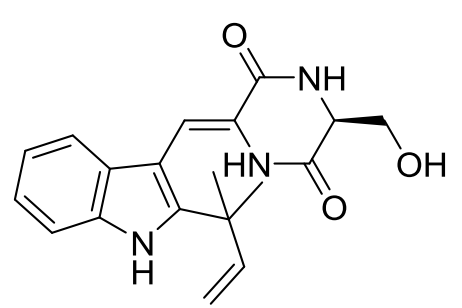

46<smiles>C=CC(C)(C)c1[nH]c2ccccc2c1/C=C1\NC(=O)C2(CC3CCC2CC3=C)OC1(C)C</smiles><smiles></smiles>

44<smiles></smiles>

45

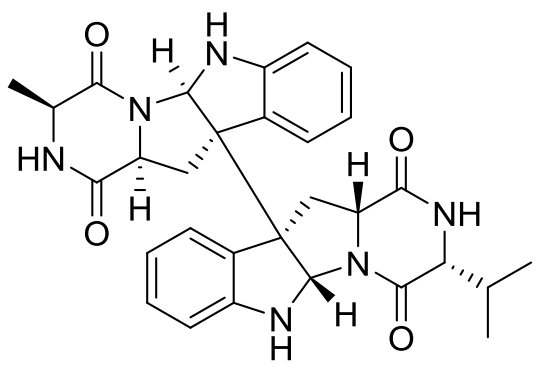

48 
Figure 5. Structures of 2,5-DKPs from marine-derived fungi of mangrove rhizosphere soil origin.
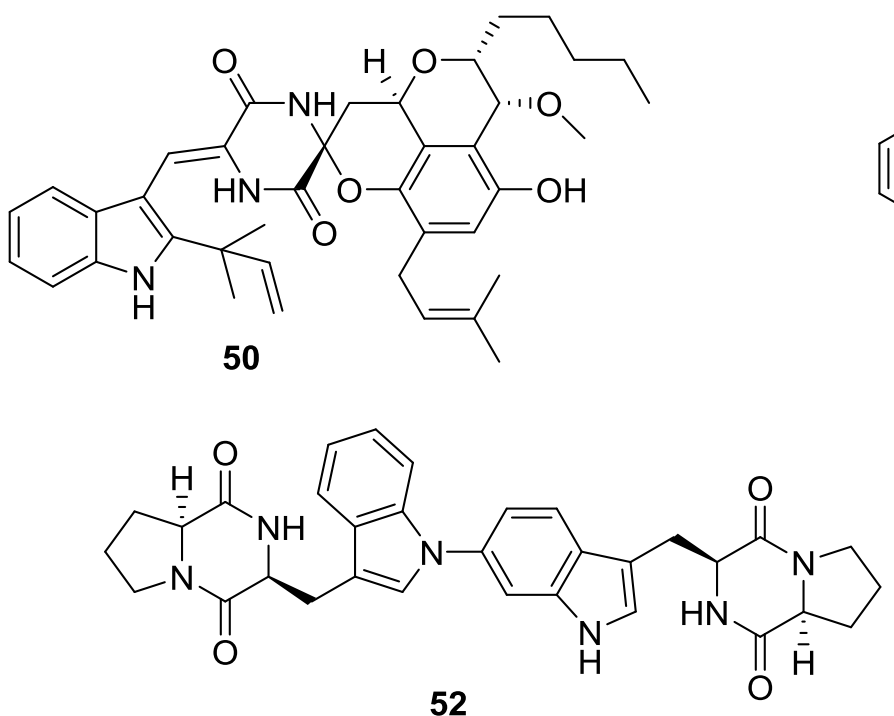

52
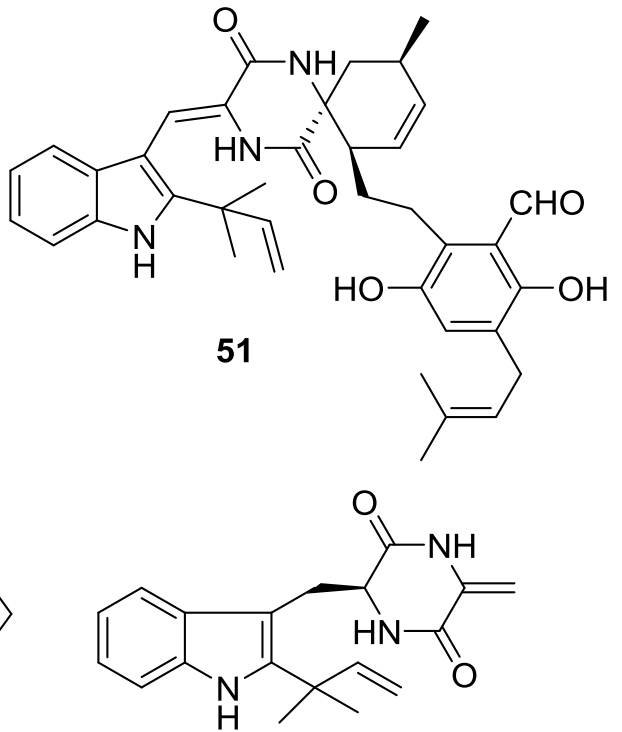

53

Figure 6. Structures of 2,5-DKPs from marine-derived fungi of mangrove origin.<smiles>CN1C(=O)C2(O)C(O)CCN2C(=O)/C1=C/c1ccccc1</smiles>

54

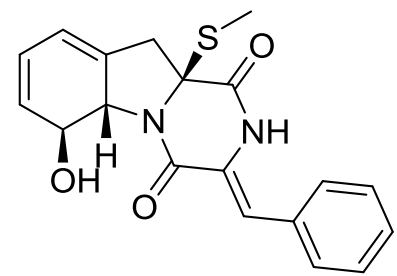

57
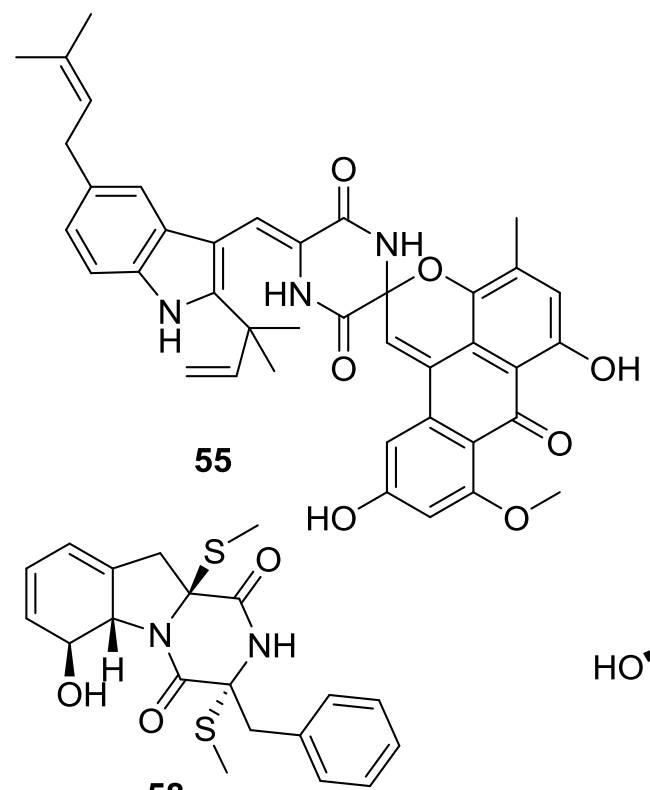

58<smiles>C=CC(C)(C)c1[nH]c2c(CC=C(C)C)cc(CC=C(C)C)cc2c1/C=C1\NC(=O)C(=O)NC1=O</smiles>

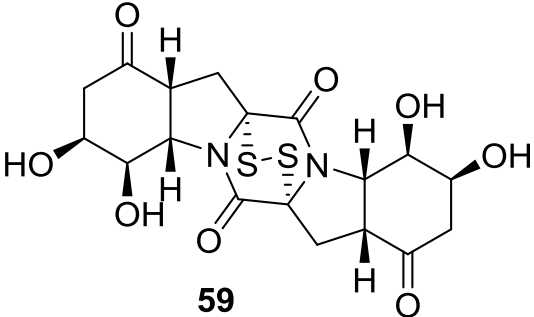

Figure 7. Structures of 2,5-DKPs from marine-derived fungi of sponge origin.

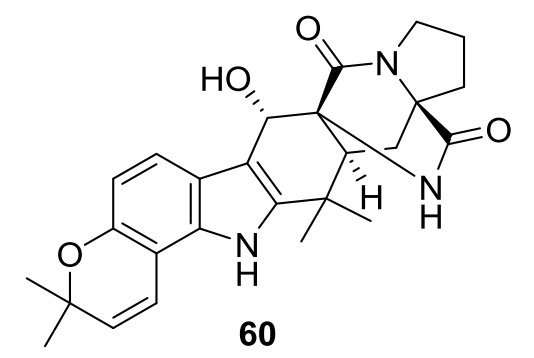

60<smiles>CC(C)[C@H]1NC(=O)/C(=C/c2c[nH]cn2)NC1=O</smiles>

61

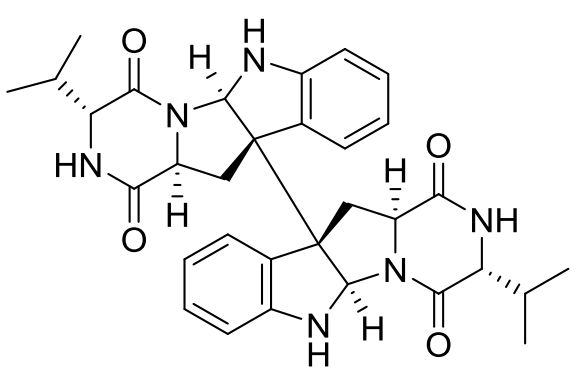

62 
Figure 8. Structures of 2,5-DKPs from marine-derived fungi of mud origin.<smiles>CC(C)=CCC/C(C)=C/COc1ccc(/C=C2/NC(=O)[C@@H](C)N(O)C2=O)cc1</smiles><smiles>COc1ccc2c3c(n(CC=C(C)C)c2c1)[C@@H](C=C(C)C)N1C(=O)[C@@H]2OCCN2C(=O)[C@]1(O)[C@H]3OC</smiles><smiles>COc1ccc2c3c([nH]c2c1)[C@@H](CC(C)(C)O)N1C(=O)C2OCCN2C(=O)[C@]1(O)[C@H]3O</smiles><smiles>COc1ccc2c3c([nH]c2c1)[C@H](C=C(C)C)N1C(=O)[C@@H]2OCCN2C(=O)[C@]1(O)C3</smiles><smiles>CC(C)=CCc1[nH]c2cc(O)ccc2c1C[C@@H]1NC(=O)[C@@H]2CCCN2C1=O</smiles><smiles>CS[C@]1(CO)NC(=O)[C@]2(CC3=CC=C[C@H](O)[C@H]3S2)NC1=O</smiles>

Figure 9. Structures of 2,5-DKPs from marine-derived fungi of mollusk origin.

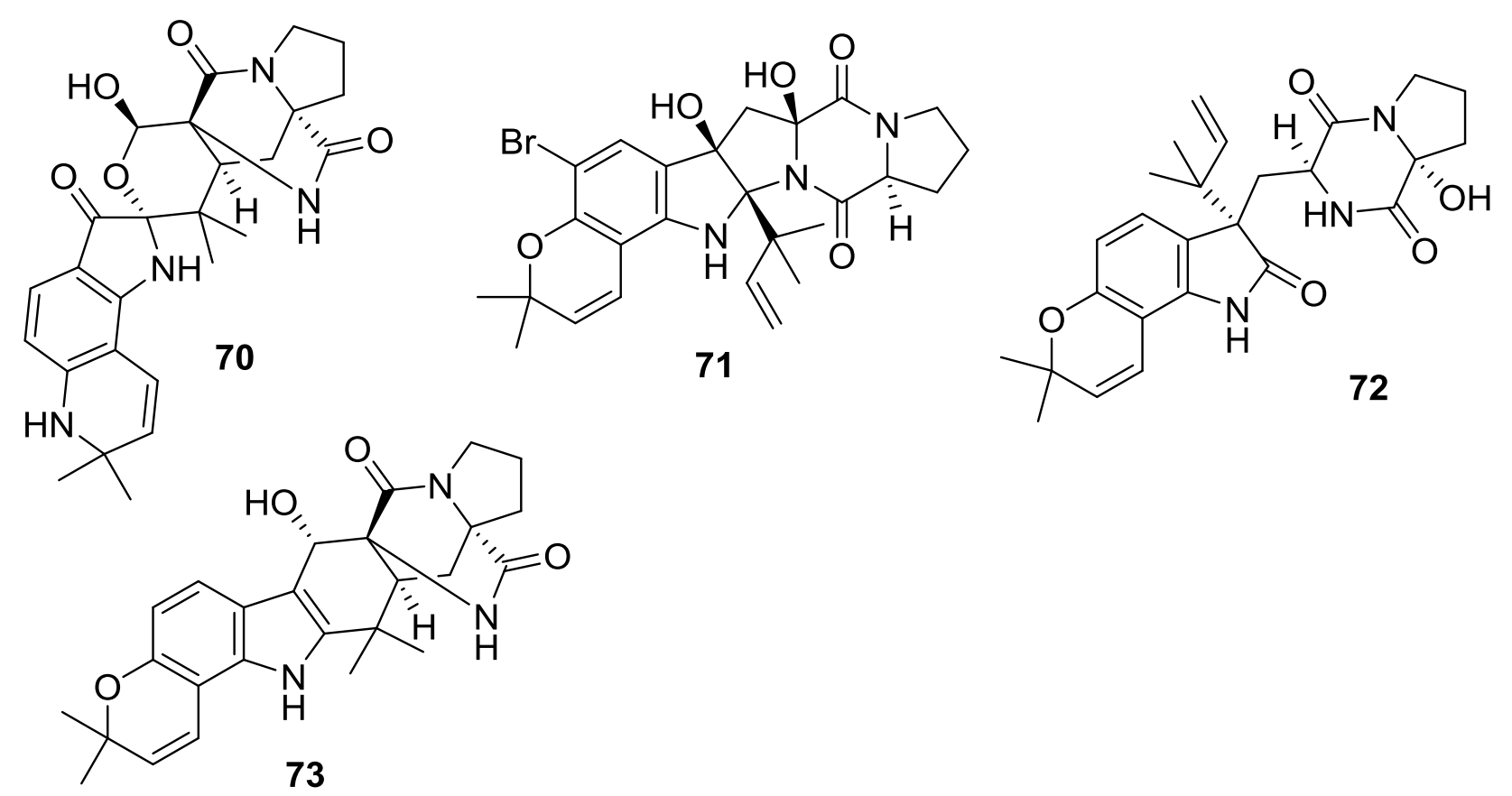


Figure 10. Structures of 2,5-DKPs from marine-derived fungi of other origins.

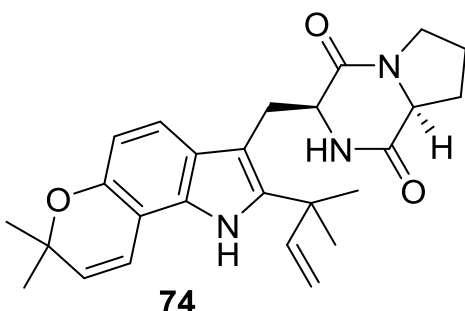

74<smiles>C=CC(C)(C)C1=Nc2c(ccc3c2C=CC(C)(C)O3)C(=O)C[C@@H]2C(=O)N3CCC[C@H]3C(=O)N12</smiles>

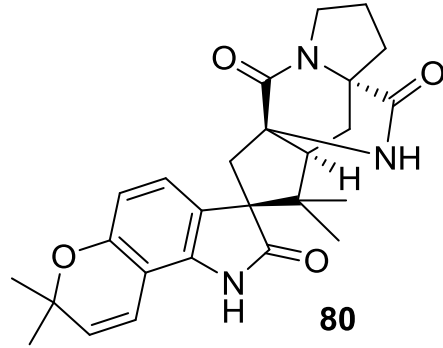<smiles>COc1ccc2c(c1)[C@]1(C(=O)N2)[C@@H](O)[C@H](O)C(=O)N2C(=O)[C@H]3CCCN3C(=O)[C@H]21</smiles><smiles>C=CC(C)(C)c1[nH]c2c3c(ccc2c1C[C@@H]1NC(=O)[C@@H]2CCCN2C1=O)C(=O)[C@H](O)[C@@H](O)C(C)(C)O3</smiles><smiles>C=C(C)C12CCC3(CCCN1C(=O)CC3C(=O)c1ccc3c(c1N)C=CC(C)(C)O3)C2=O</smiles>

78<smiles>[R][R]=CC(C)(C[R])CC#C</smiles><smiles>O=C1N[C@H](Cc2c[nH]c3ccccc23)C(=O)N/C1=C\c1c[nH]cn1</smiles><smiles>C=CC(C)(C)c1[nH]c2c3c(ccc2c1C[C@@H]1NC(=O)[C@@H]2CCCN2C1=O)C(=O)[C@H](O)[C@@H](O)C(C)(C)O3</smiles><smiles>C=CC(C)(C)C1(CC2NC(=O)C3(O)CCCN2C3=O)C(=O)Nc2c1ccc1c2C=CC(C)(C)O1</smiles>

Table 3. 2,5-DKPs from marine-derived fungi of sediment origin.

\begin{tabular}{ccccc}
\hline Number & Name & Bioactivity & Source & Reference \\
\hline $\mathbf{1 5}$ & Alternarosin A & - & Alternaria raphani & {$[11]$} \\
$\mathbf{1 6}$ & Not given & - & fumigatus & {$[12]$} \\
$\mathbf{1 7}$ & Roquefortine F & Cytotoxic activity & Penicillium sp. & {$[13]$} \\
$\mathbf{1 8}$ & Roquefortine G & Cytotoxic activity & Penicillium sp. & {$[13]$} \\
$\mathbf{1 9}$ & Plectoshpaeroic acid A & Inhibitor of indoleamine & Plectosphaerella & {$[14]$} \\
& & 2,3 -dioxygenase (IDO) & cucumerina & \\
$\mathbf{2 0}$ & Plectoshpaeroic acid B & Inhibitor of indoleamine & P. cucumerina & {$[14]$} \\
& & 2,3-dioxygenase (IDO) & & \\
$\mathbf{2 1}$ & Plectoshpaeroic acid C & Inhibitor of indoleamine & P.cucumerina & {$[14]$} \\
$\mathbf{2 2}$ & Not given & 2,3-dioxygenase (IDO) & A. fumigatus & {$[15]$} \\
$\mathbf{2 3}$ & Not given & - & A. fumigatus & {$[15]$} \\
$\mathbf{2 4}$ & Not given & - & A. fumigatus & {$[15]$} \\
$\mathbf{2 5}$ & Azonazine & Anti-inflammatory activity & A. insulicola & {$[16]$} \\
$\mathbf{2 6}$ & Roquefortine H & - & Penicillium sp. & {$[17]$} \\
$\mathbf{2 7}$ & Roquefortine I & - & Penicillium sp. & {$[17]$} \\
\hline
\end{tabular}


Table 3. Cont.

\begin{tabular}{|c|c|c|c|c|}
\hline 28 & Deoxyapoaranotin & - & A. versicolor & {$[18]$} \\
\hline 29 & Protuboxepin A & - & Aspergillus sp. & {$[19]$} \\
\hline 30 & Protuboxepin B & - & Aspergillus sp. & [19] \\
\hline 31 & Luteoalbusin A & $\begin{array}{c}\text { Potent cytotoxins against } \\
\text { several HTCLs }\end{array}$ & A. luteoaltus & {$[20]$} \\
\hline 32 & Luteoalbusin B & $\begin{array}{c}\text { Potent cytotoxins against } \\
\text { several HTCLs }\end{array}$ & A. luteoaltus & {$[20]$} \\
\hline 33 & Brevianamide $\mathrm{S}$ & $\begin{array}{l}\text { Significant antibacterial } \\
\text { activity against Bacille } \\
\text { Calmette-Guerin (BCG) }\end{array}$ & A. versicolor & {$[21]$} \\
\hline 34 & Brevianamide $\mathrm{T}$ & - & A. versicolor & {$[21]$} \\
\hline 35 & Brevianamide $\mathrm{U}$ & - & A. versicolor & {$[21]$} \\
\hline 36 & Brevianamide $\mathrm{V}$ & - & A. versicolor & {$[21]$} \\
\hline 37 & $\begin{array}{c}\text { Bis(dethio)-10a-methylthio- } \\
\text { 3a-deoxy-3,3a- } \\
\text { didehydrogliotoxin }\end{array}$ & - & Penicillium sp. & {$[22]$} \\
\hline 38 & $\begin{array}{c}\text { 6-Deoxy-5a,6- } \\
\text { didehydrogliotoxin }\end{array}$ & - & Penicillium sp. & {$[22]$} \\
\hline 39 & 5-Chlorosclerotiamide & - & A. westerdijkiae & {$[23]$} \\
\hline 40 & 10-Epi-sclerotiamide & - & A. westerdijkiae & [23] \\
\hline 41 & Nocazine D & - & Nocardiopsis alba & {$[24]$} \\
\hline 42 & Nocazine E & - & N. alba & [24] \\
\hline
\end{tabular}

Table 4. 2,5-DKPs from marine-derived fungi of algae origin.

\begin{tabular}{ccccc}
\hline Number & Name & Bioactivity & Source & Reference \\
\hline $\mathbf{4 3}$ & Carneamide A & - & A. carneus & {$[25]$} \\
$\mathbf{4 4}$ & Carneamide B & - & A. carneus & {$[25]$} \\
$\mathbf{4 5}$ & Carneamide C & - & A. carneus & {$[25]$} \\
$\mathbf{4 6}$ & Cristatumin A & Moderate activity against E. coli & E. cristatum & {$[26]$} \\
$\mathbf{4 7}$ & Cristatumin B & Moderate lethal activity against & E. cristatum & {$[26]$} \\
$\mathbf{4 8}$ & Cristatumin C & brine shrimp & E.cristatum & {$[26]$} \\
$\mathbf{4 9}$ & 9 - $O$-2(2,3-dimethylbut- & - & A. versicolor & {$[27]$} \\
\hline
\end{tabular}

Table 5. 2,5-DKPs from marine-derived fungi of mangrove rhizosphere soil origin.

\begin{tabular}{ccccc}
\hline Number & Name & Bioactivity & Source & Reference \\
\hline $\mathbf{5 0}$ & Effusin A & - & A. effuses & {$[28]$} \\
\hline \multirow{5}{*}{$\mathbf{2 1}$} & Dihydrocryptoechinuline D & $\begin{array}{c}\text { Potent activity on P388 cells with an } \\
\text { IC }_{50} \text { value of } 1.83 \mu \mathrm{M}\end{array}$ & A. effuses & {$[28]$} \\
\hline \multirow{2}{*}{5} & Aspergilazine A & Weak activity against influenza & A. taichungensis & {$[29]$} \\
\hline \multirow{2}{*}{$\mathbf{5 3}$} & Dihydroneochinulin B & Weak activity against BEL-7402 and & A. effuses & {$[30]$} \\
\hline
\end{tabular}


Table 6. 2,5-DKPs from marine-derived fungi of mangrove origin.

\begin{tabular}{|c|c|c|c|c|}
\hline Number & Name & Bioactivity & Source & Reference \\
\hline 54 & $\begin{array}{c}\text { 3-Benzylidene-8,8a- } \\
\text { dihydroxy-2-methyl- } \\
\text { hexahydro-pyrrolo[1,2-a] } \\
\text { pyrazine-1,4-dione }\end{array}$ & - & unidentified & {$[31]$} \\
\hline 55 & 7-O-methylvariecoloride A & - & E. rubrum & [32] \\
\hline 56 & $\begin{array}{c}\text { 12-Demethyl-12-oxo- } \\
\text { eurotechinulin B }\end{array}$ & Displayed cytotoxic activities & E. rubrum & {$[33]$} \\
\hline 57 & Phomazine A & - & Phoma sp. & {$[34]$} \\
\hline 58 & Phomazine B & $\begin{array}{c}\text { Moderate cytotoxicities against the HL-60, } \\
\text { HCT-116, K562, MGC-803 and A549 } \\
\text { cell lines }\end{array}$ & Phoma sp. & {$[34]$} \\
\hline 59 & Phomazine C & - & Phoma sp. & [34] \\
\hline
\end{tabular}

Table 7. 2,5-DKPs from marine-derived fungi of sponge origin.

\begin{tabular}{ccccc}
\hline Number & Name & Bioactivity & Source & Reference \\
\hline $\mathbf{6 0}$ & 21-Hydroxystephacidin & - & A. ostianus & {$[35]$} \\
$\mathbf{6 1}$ & Pre-aurantiamine & - & A. aculeatus & {$[36]$} \\
$\mathbf{6 2}$ & Eurocristatine & - & E. cristatum & {$[37]$} \\
\hline
\end{tabular}

Table 8. 2,5-DKPs from marine-derived fungi of mud origin.

\begin{tabular}{|c|c|c|c|c|}
\hline Number & Name & Bioactivity & Source & Reference \\
\hline 63 & Gliocladride A & $\begin{array}{l}\text { Cytotoxic activity against HL-60, } \\
\text { U937 and T47D with } \text { IC }_{50} \text { values } \\
\text { form } 12.80 \mu \mathrm{g} / \mathrm{mL} \text { to } 42.80 \mu \mathrm{g} / \mathrm{mL}\end{array}$ & $\begin{array}{l}\text { Gliocldium } \\
\text { sp. }\end{array}$ & [38] \\
\hline 64 & Gliocladride B & $\begin{array}{l}\text { Cytotoxic activity against HL-60, } \\
\text { U937 and T47D with IC } 50 \text { values } \\
\text { form } 11.60 \mu \mathrm{g} / \mathrm{mL} \text { to } 52.83 \mu \mathrm{g} / \mathrm{mL}\end{array}$ & $\begin{array}{l}\text { Gliocldium } \\
\quad \text { sp. }\end{array}$ & {$[38]$} \\
\hline 65 & Prenylcyclotryprostatin B & $\begin{array}{l}\text { Most potent activities against both } \\
\text { U937 and PC-3 cell lines }\end{array}$ & A. fumigatus & [39] \\
\hline 66 & 20-Hydroxycyclotryprostatin B & $\begin{array}{l}\text { Most potent activities against both } \\
\text { U937 and PC-3 cell lines }\end{array}$ & $\begin{array}{l}\text { A. fumigatus, } \\
\text { A. sydowii }\end{array}$ & [39-41] \\
\hline 67 & 9-Hydroxyfumitremorgin C & $\begin{array}{l}\text { Most potent activities against both } \\
\text { U937 and PC-3 cell lines }\end{array}$ & A. fumigatus & [39] \\
\hline 68 & 6-Hydroxytryprostatin B & $\begin{array}{l}\text { Most potent activities against both } \\
\text { U937 and PC-3 cell lines }\end{array}$ & A. fumigatus & [39] \\
\hline 69 & Spirogliotoxin & $\begin{array}{l}\text { Most potent activities against both } \\
\text { U937 and PC- } 3 \text { cell lines }\end{array}$ & A. fumigatus & [39] \\
\hline
\end{tabular}


Table 9. 2,5-DKPs from marine-derived fungi of mollusk origin.

\begin{tabular}{ccccc}
\hline Number & Name & Bioactivity & Source & Reference \\
\hline $\mathbf{7 0}$ & Notoamide O & - & Aspergillus $\mathrm{sp}$. & {$[42]$} \\
$\mathbf{7 1}$ & Notoamide P & - & Aspergillus $\mathrm{sp}$. & {$[42]$} \\
$\mathbf{7 2}$ & Notoamide Q & - & Aspergillus $\mathrm{sp}$. & {$[42]$} \\
$\mathbf{7 3}$ & Notoamide R & - & Aspergillus $\mathrm{sp}$. & {$[42]$} \\
\hline
\end{tabular}

Table 10. 2,5-DKPs from marine-derived fungi of other origins.

\begin{tabular}{ccccc}
\hline Number & Name & Bioactivity & Source & Reference \\
\hline $\mathbf{7 4}$ & Notamide E & - & Aspergillus sp. & {$[43,44]$} \\
$\mathbf{7 5}$ & Notamide E2 & - & Aspergillus sp. & {$[45]$} \\
$\mathbf{7 6}$ & Notamide E3 & - & Aspergillus sp. & {$[45]$} \\
$\mathbf{7 7}$ & Notamide E4 & - & Aspergillus sp. & {$[45]$} \\
$\mathbf{7 8}$ & Notamide L & - & Aspergillus sp. & {$[46]$} \\
$\mathbf{7 9}$ & Notamide M & - & Aspergillus sp. & {$[46]$} \\
$\mathbf{8 0}$ & Notamide N & - & Aspergillus sp. & {$[46]$} \\
$\mathbf{8 1}$ & Cyclomarazine M & - & Salinispora arenicola & {$[47]$} \\
$\mathbf{8 2}$ & Cyclomarazine P & - & S. arenicola & {$[47]$} \\
$\mathbf{8 3}$ & Notoamide S & - & Aspergillus sp. & {$[48,49]$} \\
$\mathbf{8 4}$ & Spirotryprostatin F & Stimulatory phytoregulatory activity & A. fumigatus & {$[50]$} \\
$\mathbf{8 5}$ & Penilloid A & - & Penicillium sp. & {$[51]$} \\
\hline
\end{tabular}

\subsubsection{Fungi from Sediment Origin}

An Alternaria raphani (sediment, sea salt field, Qingdao, China) yielded a DKP alkaloid, alternarosin A (15) [11]. A gliotoxin analogue (16) was isolated from the Aspergillus fumigatus (sediment, Jiaozhou Bay, Qingdao, China) [12], and the configuration assignment of $\mathbf{1 6}$ was then revised [12]. The DKP alkaloids, roquefortines F (17) and G (18) were isolated from the Penicillium sp. (sediment, $5080 \mathrm{~m}$, location not given), and their biogenetic relationships were postulated in a plausible pathway (Scheme 2) [13]. The DKP alkaloids, plectoshpaeroic acids A-C (19-21) were isolated from the Plectosphaerella cucumerina (sediments, Barkley Sound, British Columbia) and identified as inhibitors of indoleamine 2,3-dioxygenase (IDO), an enzyme catalyzing the conversion of the essential amino acid L-tryptophan to $N$-formylkynurenine [14]. DKPs (22-24) were isolated from the A. fumigatus (sediment, Jiaozhou Bay, China) [15]. The fermentation of A. insulicola (sediment, Hawaii) resulted in the discovery of a hexacyclic dipeptide azonazine (25), which exhibited an anti-inflammatory activity through the inhibition of NF- $\kappa B$ luciferase and nitrite production [16]. Two DKP derivatives, roquefortines $\mathrm{H}$ (26) and I (27) were isolated from the Penicillium sp. (deep ocean sediment, unspecified location) [17]. The DKP disulfide deoxyapoaranotin (28) from the A. versicolor (sediment, East Sea, Korea) was reported to be cytotoxic, and it showed an induced apoptotic activity against HCT-116 [18]. An Aspergillus sp. (sediment, Dadaepo Beach, Busan, Korea) gave two DKP-type alkaloids (29) and (30) [19]. Two indole DKPs, luteoalbusins A (31) and B (32) were isolated from the Acrostalagmus luteoaltus (deep sea sediment, South China Sea), and they were identified as potent cytotoxins against several HTCLs [20]. The dimeric DKP brevianamide S (33) and the monomeric brevianamides T-V (34-36) were isolated from the A. versicolor (sediment, Bohai Sea, 
China). A plausible biosynthetic relationship linking these brevianamide derivatives through a sequence of oxidative transformations was described. Brevianamide $S$ (33) showed a selective activity against the Bacille Calmette-Guérin (BCG) strain of Mycobacterium bovis, which suggested a new mechanism of the action with potential to be an antitubercular drug lead [21]. The isolation of the Penicillium sp. (deep sea sediment, Suruga Bay, Japan) yielded two gliotoxin-related compounds, including 37 and 38. Compound (38) was shown to be cytotoxic to P388 cells [22]. Two prenylated indole alkaloids, 5-chlorosclerotiamide (39) and 10-epi-sclerotiamide (40) were isolated from the deep-sea-derived fungus A. westerdijkiae DFFSCS013 (sediment, South China Sea) [23]. Two DKP enantiomers, nocazines D (41) and E (42) were isolated from the Nocardiopsis alba SCSIO 03039 (sediment, Indian Ocean) [24].

Scheme 2. Postulated biosynthetic pathway for 17 and 18 [13].

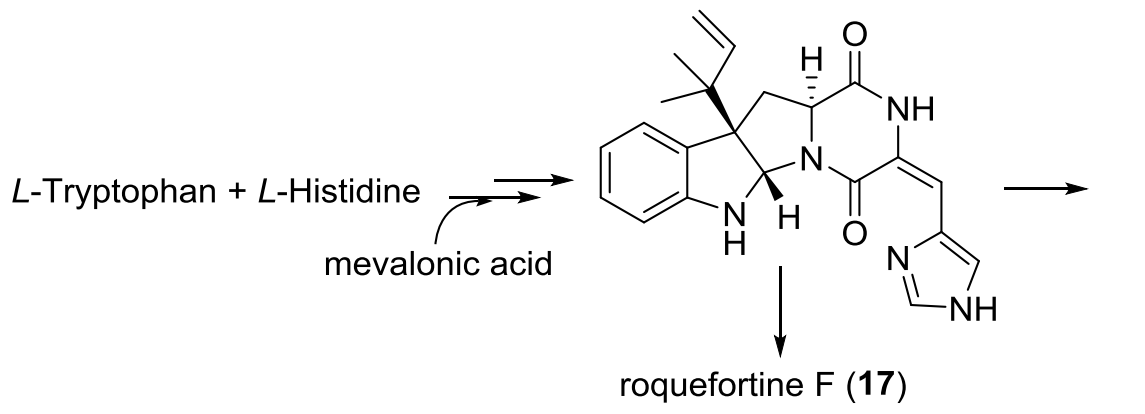<smiles>C=CC(C)(C)C1(C(C)(C)C)CN2C(=O)/C(=C\c3c[nH]cn3)NC(=O)[C@]2(O)C1</smiles><smiles>C=CC(C)(C)C12C[C@]3(OC)C(=O)N/C(=C/c4c[nH]cn4)C(=O)N3[C@H]1Nc1ccccc12</smiles><smiles>CC(=O)OCCCCCCCCCCCCC(=O)OCC(C)(O)CC(=O)CC(=O)O</smiles><smiles>CC(C)(O)C(=O)O</smiles><smiles>C=C(C)CC(C)=O</smiles>

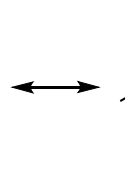
a Michael addition roquefortine $\mathrm{G}(\mathbf{1 8})$

\subsubsection{Fungi from Algae Origin}

An A. carneus (brown alga Laminaria sachalinensis, Kunachir Is., Russia) was the source of the prenylated indole alkaloids, carneamides $\mathrm{A}-\mathrm{C}(\mathbf{4 3}-\mathbf{4 5})$, for which a possible biosynthetic pathway was proposed (Scheme 3) [25]. The endophytic fungus Eurotium cristatum (brown alga Sargassum thunbergii, location unspecified) was the source of the indole alkaloids, cristatumins A-C (46-48) [26]. 9E-O-2(2,3-dimethylbut-3-enyl)brevianamide $\mathrm{Q}(\mathbf{4 9})$ was isolated from the endophytic fungus A. versicolor (brown alga $S$. thunbergii, Pingtan Is., China) [27].

\subsubsection{Fungi from Mangrove Rhizosphere Soil Origin}

The racemic spiroalkaloids, effusin A and dihydrocryptoechinulin D (shown here as one of the enantiomers, effusin A (50) and dihydrocryptoechinuline D (51), respectively) were obtained from the A. effuses (mangrove rhizosphere soil, Fujian, China). The racemates were subsequently resolved, and 
their absolute configurations were determined by the solution time dependent density function theory (TDDFT) and electronic CD (ECD) calculations. Effusin A (50) contains a spirobicyclic $N, O$-acetal moiety, which could be obtained by a domino ring-closure reaction between the substituted salicylaldehyde moiety in aspergin and the eneamide moiety of the DKP unit in neoechinulin B. In contrast, an enzyme-catalyzed regiospecific [4 +2] Diels-Alder reaction produces the spirobicycle of dihydrocryptoechinuline $\mathrm{D}$ (51). The racemate of dihydrocryptoechinulin D inhibited the growth of P388 cells, and the (12R,28S,31S)-enantiomer 51 showed a selective, moderate inhibition of topoisomerase I [28]. Aspergilazine A (52), a DKP dimer consisting of two DKP units with a rare N-1 to C-6 linkage, was obtained from the A. taichungensis (mangrove root soil, Acrostichum aureum, source not given) [29]. The prenylated indole DKP alkaloid, dihydroneochinulin B (53) was isolated from the fungus $A$. effuses H1-1 (mangrove rhizosphere soil, Fujian, China) [30].

Scheme 3. Postulated biosynthetic pathway for carneamides A-C (43-45) [25].

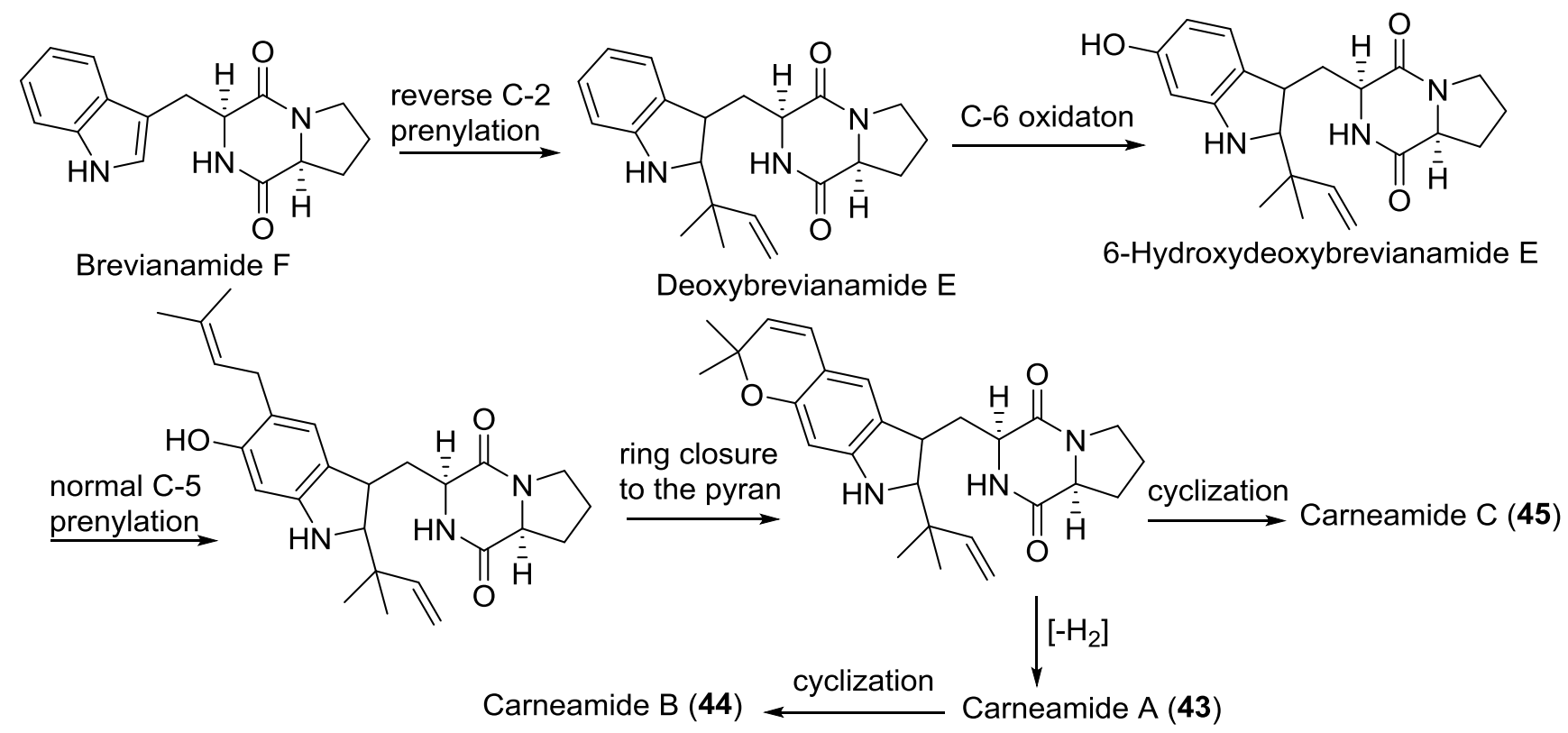

\subsubsection{Fungi from Mangrove Origin}

The amide alkaloid (54) was isolated from an unidentified endophytic fungus (mangrove, Acanthus ilicifolius, South China Sea) [31]. The spirocyclic DKP alkaloid, 7-O-methylvariecoloride A (55) was sourced from the Eurotium rubrum (stem of the mangrove Hibiscus tiliaceus, Hainan Is., China) [32]. An endophytic strain of E. rubrum, (semi-mangrove H. tiliaceus, Hainan Is., China) produced a DKP alkaloid, 12-demethyl-12-oxo-eurotechinulin B (56) [33]. Furthermore, three thiodiketopiperazines, phomazines A-C (57-59) were isolated from an endophytic fungus Phoma sp. OUCMDZ-1847 (mangrove Kandelia candel, Wenchang, Hainan Province, China) [34].

\subsubsection{Fungi from Sponge Origin}

A heptacyclic alkaloid, 21-hydroxystephacidin (60) was isolated from the A. ostianus (unidentified sponge, Pohnpei) [35]. Pre-aurantiamine (61) was isolated from the A. aculeatus (sponge Stylissa flabelliformis, Phi Phi Is., Krabi Province, Thailand) [36]. The DKP dimer, eurocristatine (70) 
was isolated from the endophytic fungus Eurotium cristatum (sponge Mycale sp., Wonnapa Beach, Bangsaen, Thailand) [37].

\subsubsection{Fungi from Mud Origin}

Two piperazine-2,5-dione derivatives, gliocladrides A $(63)$ and $\mathrm{B}(\mathbf{6 4})$ were isolated from the Gliocldium sp. (sea mud, Rushan, China) [38]. Five DKPs, prenylcyclotryprostatin B (65), 20-hydroxycyclotryprostatin B (66), 9-hydroxyfumitremorgin C (67), 6-hydroxytryprostatin B (68) and spirogliotoxin (69) were isolated from the A. fumigatus (intertidal mud, Yingkou, China). Prenylcyclotryprostatin B and 9-hydroxyfumitremorgin $\mathrm{C}$ were shown to be moderate inhibitors of human leukemic monocyte lymphoma (U937) cells [39]. 20-Hydroxycyclotryprostatin B was also isolated from two other sources in 2012, firstly from the A. sydowii (gorgonian coral Verrucella umbraculum, Sanya, Hainan province, China) as cyclotryprostatin E [40] and secondly, from a

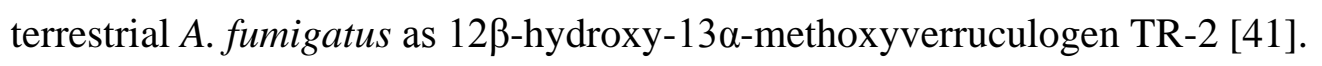

\subsubsection{Fungi from Mollusk Origin}

The fermentation of Aspergillus sp. (mollusk Mytilis edulis galloprovincialis, Noto Penninsula, Sea of Japan) yielded the notoamides $\mathrm{O}-\mathrm{R}$ (70-73). Notoamide $\mathrm{O}$ (70) is noteworthy as the compound consists of a novel hemiacetal/hemiaminal ether moiety, which represents an unusual branch point for the oxidative modification of other members in the family of the prenylated indole alkaloids in the biogenetic pathway (Scheme 4) [42]. The structure of notoamide Q (72) has been revised [42]. The whole genome sequencing of Aspergillus MF 297-2 (mollusk M. edulis galloprovincialis, Noto Penninsula, Japan Sea) [52] led to the identification and characterization of the biosynthetic gene cluster for stephacidins [53] and the notoamide alkaloids [43,52].

Scheme 4. Postulated biosynthetic pathway for 70 [42].

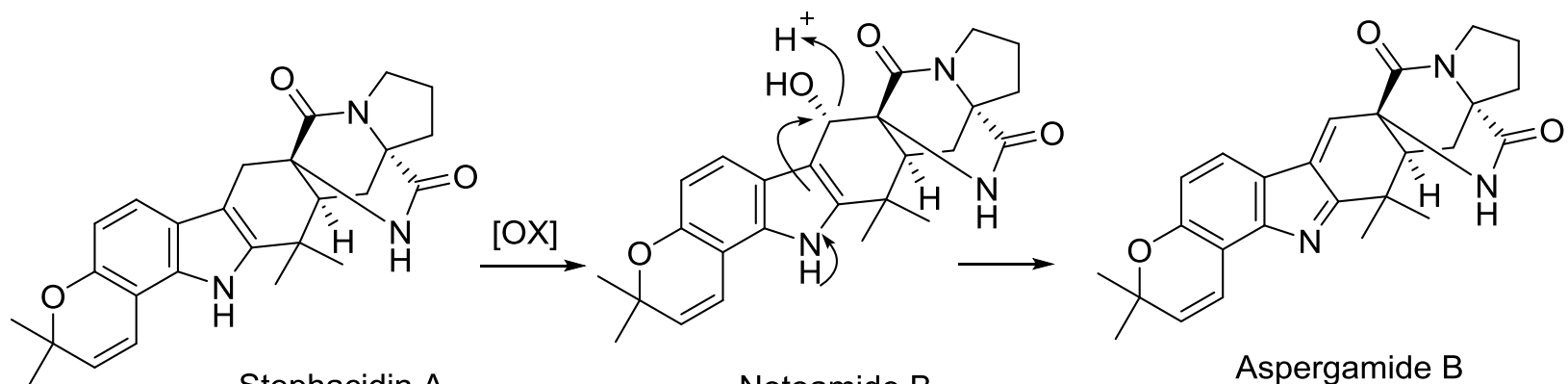

Stephacidin A

Notoamide B

Aspergamide B

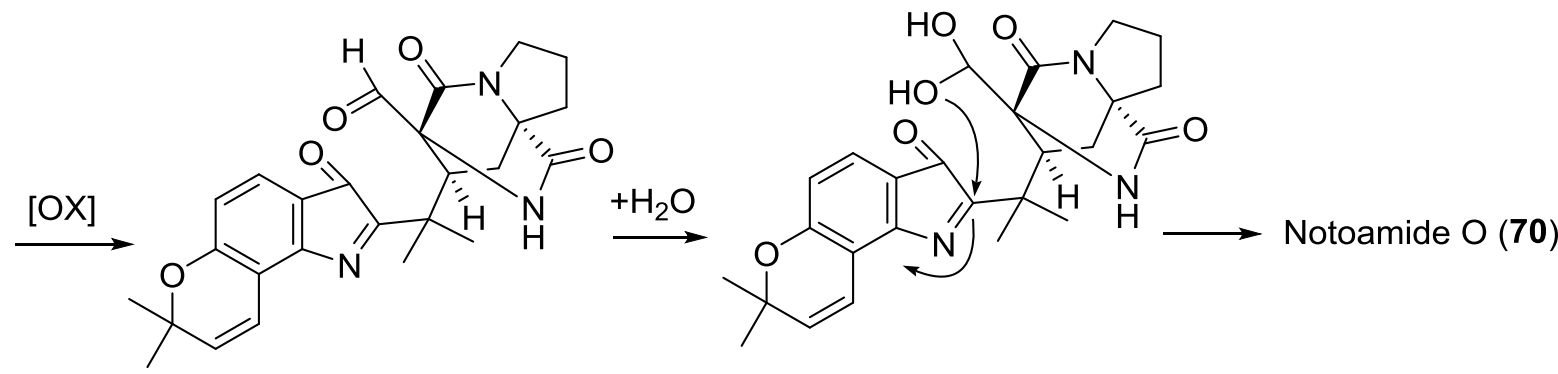




\subsubsection{Fungi from Other Origins}

Notamide E (74) was isolated from the culture of Aspergillus sp. (mussel Mytilus edulis, Noto Peninsula, Sea of Japan) [43]. The compound (74) has been synthesized prior to its isolation from the natural source, and it was proposed [44] to be an advanced precursor to notoamides A-D. The biosynthetic studies of the producing organism indicated that notoamide E (74) was a short-lived metabolite. The feeding experiments utilizing synthetic, ${ }^{13} \mathrm{C}$-labelled (74) demonstrated the incorporation of notoamide $\mathrm{E}$ into notoamides C [43], D [45] and 3-epi-notamide C [44]. These studies also produced three minor alkaloids, notamides E2-E4 (75-77) [45]. Further investigation of the same culture of Aspergillus that yielded notamides A-D [45] led to the isolations of notamides L-N (78-80) [46]. In a direct and targeted gene manipulation experiment, the provision of synthetic $N$-alkyl tryptophan to a prenyltransferase-deficient mutant of a cyclomarin/cyclomarazine-producing $S$. arenicola led to the discovery of some novel derivatives, cyclomarazines $\mathrm{M}$ (81) and P (82) [47]. Two central pathway enzymes, which catalyzed both the normal and reverse prenyltransfer reactions, were characterized. The study also established the early steps of the biosynthetic procedure of prenylated indole alkaloid structure, including the production of notoamide $S(\mathbf{8 3})$ [48]. Notoamide $S(\mathbf{8 3})$ has been synthesized via $\mathrm{N}$-Fmoc proline coupling with a 6-hydroxy-7-prenyl-2-reverse prenyl tryptophan derivative [49]. The spirocyclic DKP alkaloid, spirotryprostatin F (84) was isolated from the A. fumigatus (soft coral Sinularia sp., Kunashir Is., Kuril Islands), and it showed a stimulatory phytoregulatory activity in the study [50]. An indolyl DKP compound, penilloid A (85) was isolated from two marine derived fungi Penicillium sp. and A. sydowii [51].

\section{Sponges}

Three 2,5-DKPs (86-88) have been isolated from sponges (Figure 11). A Callyspongia species (Haina Is., China) yielded callyspongidipeptide A (86) [54]. DKP 87 was obtained from Stelletta sp. (Jamieson Reef, Bonaparte Archipelago, Australia), and it was proposed to be the product of an enzymatically controlled condensation reaction between D-isoleucine and 4-S-hydroxy-D-proline [55]. A Callyspongia sp. (Haina Is., China) yielded callysponine A (88) [56].

Figure 11. Structures of 2,5-DKPs from marine sponges.

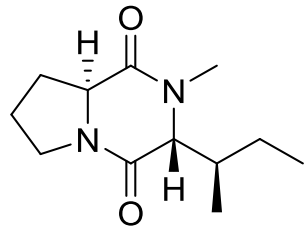

86

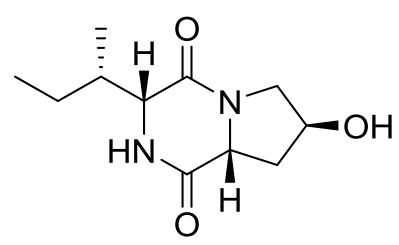

87

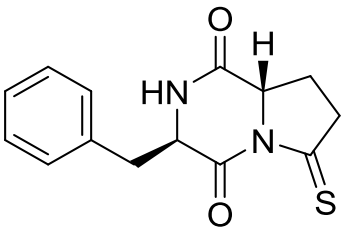

88

\section{Gorgonian}

The isolation of the gorgonian Menella kanisa collected from Beibu Gulf led to the identification of menazepine A (89) (Figure 12) [57]. 
Figure 12. Structure of 2,5-DKP from gorgonian.

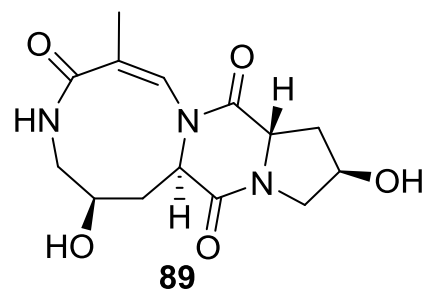

\section{Red Algae}

A collection of Symphyocladia latiuscula (Qingdao, Shandong Province, China) provided a bromophenol coupled to a DKP core structure (90) (Figure 13) [58].

Figure 13. Structure of 2,5-DKP from red algae.

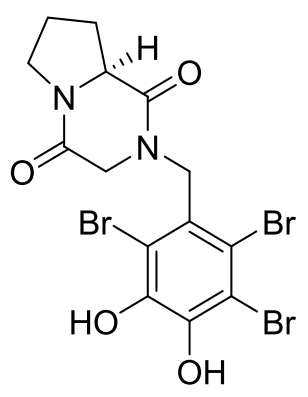

90

\section{Conclusions}

2,5-DKPs are ubiquitous in nature. They have previously been isolated from bacteria, fungi, marine invertebrates and higher organisms [1,2]. Although these DPK derivatives have been known since the early 20th century, they only recently draw significant interests because of the diverse range of their biological activities [1], including the disruption of the biofilm formation through modulation of bacterial quorum sensing and their role in an interkingdom cell-cell signaling [42]. The increasing numbers of naturally occurring bioactive 2,5-DKPs have been obtained from various marine organisms, and the studies on these 2,5-DKPs have been the focus of many recent studies because of their potent biological activities. To date, more than 200 2,5-DKPs have been isolated from a diverse range of marine organisms, particularly marine microorganisms. Some of these 2,5-DKPs exhibited various bioactivities, such as cytotoxicity on cancer cell lines, anti-microbial and anti-inflammatory properties [1]. From 2009 to the first half-year of 2014, the main natural source of DKPs isolated from marine organisms is marine microorganisms, accounting for $94 \%$ (Figure 14). Many studies have performed on these bacteria, fungi and actinomycete that produced 2,5-DKPs, and these marine microorganisms were isolated from sediments, algae, mangrove, sponges and mud (Figure 15). It was indicated that $51 \%$ of the studied microorganisms were isolated from sediments as shown in Figure 15. Interestingly, the marine-derived fungi accounted for the largest part (84\%) of total 2,5-DKPs that were isolated from marine microorganisms (Figure 16). The interest in natural products from marine microorganisms, especially marine-derived fungi, has increased significantly in the last decade $[59,60]$, 
which has led to the discoveries of more 2,5-DKPs from the marine-derived fungi than that from other marine organisms. Consequently marine microorganisms, especially fungi can be a promising source of these bioactive 2,5-DKPs. The discovery of these compounds from marine-derived fungi demonstrates that some gene clusters in fungi may have the ability to produce structurally diverse DKPs by the biosynthetic pathways, which may need further investigations.

Figure 14. The distribution of 2,5-DKPs reported from marine organisms.
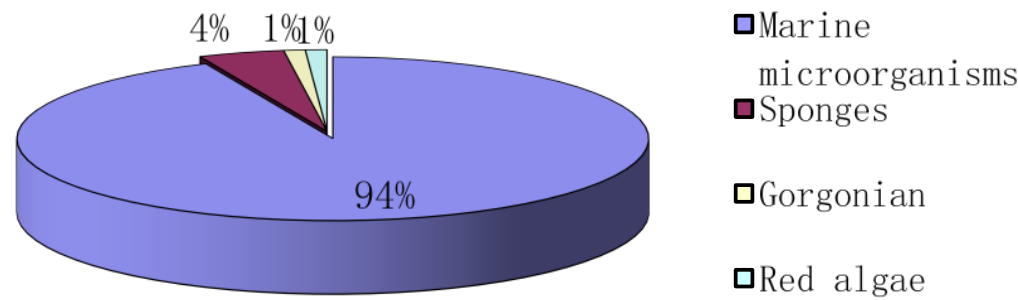

Figure 15. Origin of the microorganisms.

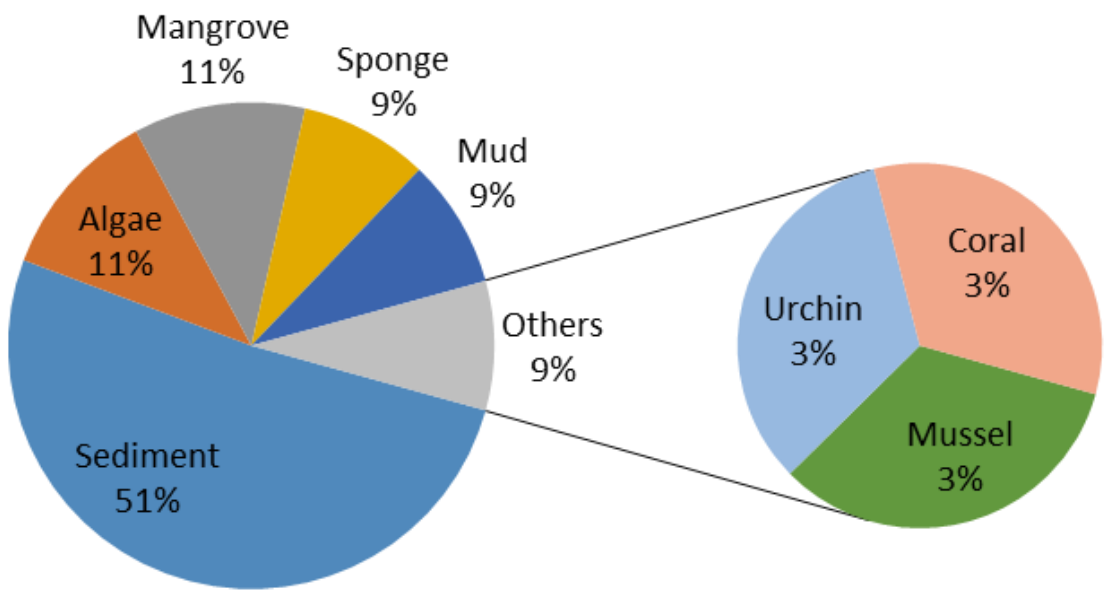

Figure 16. The distribution of 2,5-DKPs reported from marine-derived microorganisms.

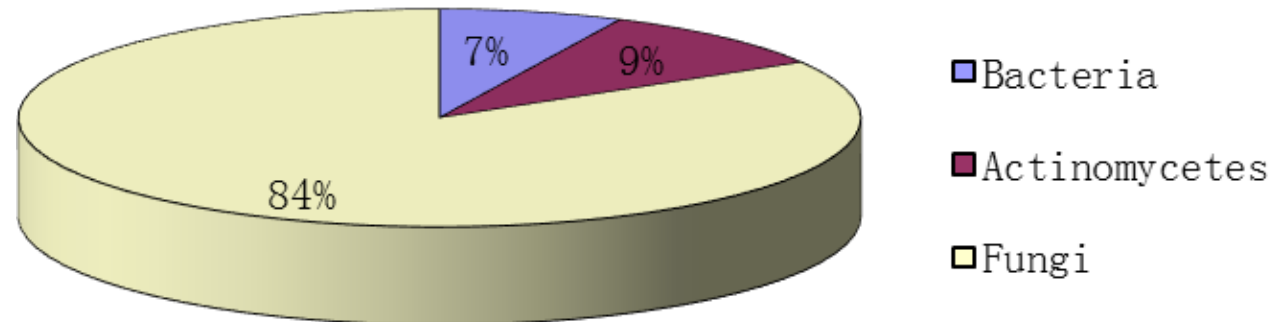

\section{Acknowledgments}

This study was supported by grants from the National Natural Science Foundation of China (No. 31100260, 81260480), the Natural Science Foundation of Guangxi (2012GXNSFAA053160, 2012GXNSFEA053001), the Knowledge Innovation Program of Chinese Academy of Sciences (KSCX2-EW-J-28), the Program of Guangzhou City (No. 12C14061559), the Key Project of Science and Technology Department of Guangxi (143001-7), and the Cooperation Projects of SIDSSE and Sanya (2013YD73). Ri-Ming Huang acknowledges the support of a CAS Academic Visitor Fellowship 
as academic visitor at University of Bath (with Ian S Blagbrough), and thanks Heather Wyman-Pain, University of Bath, for proofreading.

\section{Author Contributions}

Ri-Ming Huang contributed in writing the manuscript. Cheng-Hai Gao conceived and designed the format of the manuscript. All the authors contributed in critical reading and discussion on the manuscript.

\section{Conflicts of Interest}

The authors declare no conflict of interest.

\section{References}

1. Huang, R.M.; Zhou, X.F.; Xu, T.H.; Yang, X.W.; Liu, Y.H. Diketopiperazines from marine organisms. Chem. Biodivers. 2010, 7, 2809-2829.

2. Borthwick, A.D. 2,5-Diketopiperazines: Synthesis, reactions, medicinal chemistry, and bioactive natural products. Chem. Rev. 2012, 112, 3641-3716.

3. Martins, M.B.; Carvalho, I. Diketopiperazines: Biological activity and synthesis. Tetrahedron 2007, 63, 9923-9932.

4. Raju, R.; Piggott, A.M.; Conte, M.; Aalbersberg, W.G.L.; Feussner, K.; Capon, R.J. Naseseazines A and B: A new dimeric diketopiperazine framework from a marine-derived actinomycete, Streptomyces sp. Org. Lett. 2009, 11, 3862-3865.

5. Fu, P.; Liu, P.P.; Qu, H.J.; Wang, Y.; Chen, D.F.; Wang, H.; Li, J.; Zhu, W.M. $\alpha$-Pyrones and diketopiperazine derivatives from the marine-derived actinomycete Nocardiopsis dassonvillei HR10-5. J. Nat. Prod. 2011, 74, 2219-2223.

6. Bryans, J.; Charlton, P.; ChicarelliRobinson, I.; Collins, M.; Faint, R.; Latham, C.; Shaw, I.; Trew, S. Inhibition of plasminogen activator inhibitor-1 activity by two diketopiperazines, XR330 and XR334 produced by Streptomyces sp. J. Antibiot. 1996, 49, 1014-1021.

7. Wang, P.; Xi, L.J.; Liu, P.P.; Wang, Y.; Wang, W.; Huang, Y.; Zhu, W.M. Diketopiperazine derivatives from the marine-derived actinomycete Streptomyces sp. FXJ7.328. Mar. Drugs 2013, 11, 1035-1049.

8. Yonezawa, K.; Yamada, K.; Kouno, I. New diketopiperazine derivatives isolated from sea urchin-derived Bacillus sp. Chem. Pharm. Bull. 2011, 59, 106-108.

9. Raju, R.; Piggott, A.M.; Huang, X.C.; Capon, R.J. Nocardioazines: A novel bridged diketopiperazine scaffold from a marine-derived bacterium inhibits P-glycoprotein. Org. Lett. 2011, 13, 2770-2773.

10. Khedr, A.I.M.; Kouno, I.; Tanaka, T.; Yamada, K. New diketopiperazine derivatives from culture broth of Staphylococcus sp. isolated from Corallina officinalis Lineaus. Heterocycles 2013, 87, 1029-1037.

11. Wang, W.L.; Wang, Y.; Tao, H.W.; Peng, X.P.; Liu, P.P.; Zhu, W.M. Cerebrosides of the halotolerant fungus Alternaria raphani isolated from a sea salt field. J. Nat. Prod. 2009, 72, 1695-1698. 
12. Zhao, W.Y.; Zhu, T.J.; Han, X.X.; Fan, G.T.; Liu, H.B.; Zhu, W.M.; Gu, Q.Q. A new gliotoxin analogue from a marine-derived fungus Aspergillus fumigatus Fres. Nat. Prod. Res. 2009, 23, 203-207; Correction 1457.

13. Du, L.; Li, D.H.; Zhu, T.J.; Cai, S.X.; Wang, F.P.; Xiao, X.; Gu, Q.Q. New alkaloids and diterpenes from a deep ocean sediment derived fungus Penicillium sp. Tetrahedron 2009, 65, 1033-1039.

14. Carr, G.; Tay, W.; Bottriell, H.; Andersen, S.K.; Mauk, A.G.; Andersen, R.J. Plectosphaeroic acids $\mathrm{A}, \mathrm{B}$, and $\mathrm{C}$, indoleamine 2,3-dioxygenase inhibitors produced in culture by a marine isolate of the fungus Plectosphaerella cucumerina. Org. Lett. 2009, 11, 2996-2999.

15. Zhao, W.Y.; Zhu, T.J.; Fan, G.T.; Liu, H.B.; Fang, Y.C.; Gu, Q.Q.; Zhu, W.M. Three new dioxopiperazine metabolites from a marine-derived fungus Aspergillus fumigatus Fres. Nat. Prod. Res. 2010, 24, 953-957.

16. Wu, Q.X.; Crews, M.S.; Draskovic, M.; Sohn, J.; Johnson, T.A.; Tenney, K.; Valeriote, F.A.; Yao, X.J.; Bjeldanes, L.F.; Crews, P. Azonazine, a novel dipeptide from a Hawaiian marine sediment-derived fungus, Aspergillus insulicola. Org. Lett. 2010, 12, 4458-4461.

17. Du, L.; Feng, T.; Zhao, B.Y.; Li, D.H.; Cai, S.X.; Zhu, T.J.; Wang, F.P.; Xiao, X.; Gu, Q.Q. Alkaloids from a deep ocean sediment-derived fungus Penicillium sp. and their antitumor activities. J. Antibiot. 2010, 63, 165-170.

18. Choi, E.J.; Park, J.S.; Kim, Y.J.; Jung, J.H.; Lee, J.K.; Kwon, H.C.; Yang, H.O. Apoptosis-inducing effect of diketopiperazine disulfides produced by Aspergillus sp. KMD 901 isolated from marine sediment on HCT116 colon cancer cell lines. J. Appl. Microbiol. 2011, 110, 304-313.

19. Lee, S.U.; Asami, Y.; Lee, D.; Jang, J.H.; Ahn, J.S.; Oh, H. Protuboxepins A and B and protubonines A and B from the marine-derived fungus Aspergillus sp. SF-5044. J. Nat. Prod. 2011, 74, 1284-1287.

20. Wang, F.Z.; Huang, Z.; Shi, X.F.; Chen, Y.C.; Zhang, W.M.; Tian, X.P.; Li, J.; Zhang, S. Cytotoxic indole diketopiperazines from the deep sea-derived fungus Acrostalagmus luteoalbus SCSIO F457. Bioorg. Med. Chem. Lett. 2012, 22, 7265-7267.

21. Song, F.H.; Liu, X.R.; Guo, H.; Ren, B.; Chen, C.X.; Piggott, A.M.; Yu, K.; Gao, H.; Wang, Q.; Liu, M.; et al. Brevianamides with antitubercular potential from a marine-derived isolate of Aspergillus versicolor. Org. Lett. 2012, 14, 4770-4773.

22. Sun, Y.; Takada, K.; Takemoto, Y.; Yoshida, M.; Nogi, Y.; Okada, S.; Matsunaga, S. Gliotoxin analogues from a marine-derived fungus, Penicillium sp., and their cytotoxic and histone methyltransferase inhibitory activities. J. Nat. Prod. 2012, 75, 111-114.

23. Peng, J.; Zhang, X.Y.; Tu, Z.C.; Xu, X.Y.; Qi, S.H. Alkaloids from the deep-sea-derived fungus Aspergillus westerdijkiae DFFSCS013. J. Nat. Prod. 2013, 76, 983-987.

24. Zhang, Q.B.; Li, S.M.; Chen, Y.C.; Tian, X.P.; Zhang, H.B.; Zhang, G.T.; Zhu, Y.G.; Zhang, S.; Zhang, W.M.; Zhang, C.S. New diketopiperazine derivatives from a deep-sea-derived Nocardiopsis alba SCSIO 03039. J. Antibiot. 2013, 66, 31-36.

25. Zhuravleva, O.I.; Afiyatullov, S.S.; Denisenko, V.A.; Ermakova, S.P.; Slinkina, N.N.; Dmitrenok, P.S.; Kim, N.Y. Secondary metabolites from a marine-derived fungus Aspergillus carneus Blochwitz. Phytochemistry 2012, 80, 123-131. 
26. Du, F.Y.; Li, X.M.; Li, C.S.; Shang, Z.; Wang, B.G. Cristatumins A-D, new indole alkaloids from the marine-derived endophytic fungus Eurotium cristatum EN-220. Bioorg. Med. Chem. Lett. 2012, 22, 4650-4653.

27. Miao, F.P.; Li, X.D.; Liu, X.H.; Cichewicz, R.H.; Ji, N.Y. Secondary metabolites from an algicolous Aspergillus versicolor strain. Mar. Drugs 2012, 10, 131-139.

28. Gao, H.Q.; Liu, W.Z.; Zhu, T.J.; Mo, X.M.; Mandi, A.; Kurtan, T.; Li, J.; Ai, J.; Gu, Q.Q.; $\mathrm{Li}$, D.H. Diketopiperazine alkaloids from a mangrove rhizosphere soil derived fungus Aspergillus effuses H1-1. Org. Biomol. Chem. 2012, 10, 9501-9506.

29. Cai, S.X.; Kong, X.L.; Wang, W.; Zhou, H.N.; Zhu, T.J.; Li, D.H.; Gu, Q.Q. Aspergilazine A, a diketopiperazine dimer with a rare $\mathrm{N}-1$ to $\mathrm{C}-6$ linkage, from a marine-derived fungus Aspergillus taichungensis. Tetrahedron Lett. 2012, 53, 2615-2617.

30. Gao, H.Q.; Zhu, T.J.; Li, D.H.; Gu, Q.Q.; Liu, W.Z. Prenylated indole diketopiperazine alkaloids from a mangrove rhizosphere soil derived fungus Aspergillus effuses H1-1. Arch. Pharm. Res. 2013, 36, 952-956.

31. Chen, Y.G.; Shao, C.L.; Huang, Z.J.; Zhang, Y.; Cai, X.L.; She, Z.G.; Zhou, S.N.; Lin, Y.C. Structure elucidation and NMR assignments for two amide alkaloids from a mangrove endophytic fungus (No. ZZF-22). Magn. Reson. Chem. 2009, 47, 92-95.

32. Li, D.L.; Li, X.M.; Proksch, P.; Wang, B.G. 7-O-Methylvariecolortide A, a new spirocyclic diketopiperazine alkaloid from a marine mangrove derived endophytic fungus, Eurotium rubrum. Nat. Prod. Commun. 2010, 5, 1583-1586.

33. Yan, H.J.; Li, X.M.; Li, C.S.; Wang, B.G. Alkaloid and anthraquinone derivatives produced by the marine-derived endophytic fungus Eurotium rubrum. Helv. Chim. Acta 2012, 95, 163-168.

34. Kong, F.D.; Wang, Y.; Liu, P.P.; Dong, T.H.; Zhu, W.M. Thiodiketopiperazines from the marine-derived fungus Phoma sp. OUCMDZ-1847. J. Nat. Prod. 2014, 77, 132-137.

35. Kito, K.; Ookura, R.; Kusumi, T.; Namikoshi, M.; Ooi, T. X-ray structures of two stephacidins, heptacyclic alkaloids from the marine-derived fungus Aspergillus ostianus. Heterocycles 2009, 78, 2101-2106.

36. Antia, B.S.; Aree, T.; Kasettrathat, C.; Wiyakrutta, S.; Ekpa, O.D.; Ekpe, U.J.; Mahidol, C.; Ruchirawat, S.; Kittakoop, P. Itaconic acid derivatives and diketopiperazine from the marine-derived fungus Aspergillus aculeatus CRI322-03. Phytochemistry 2011, 72, 816-820.

37. Gomes, N.M.; Dethoup, T.; Singburaudom, N.; Gales, L.; Silva, A.M.S.; Kijjoa, A. Eurocristatine, a new diketopiperazine dimer from the marine sponge-associated fungus Eurotium cristatum. Phytochem. Lett. 2012, 5, 717-720.

38. Yao, Y.; Tian, L.; Li, J.; Cao, J.Q.; Pei, Y.H. Cytotoxic piperazine-2,5-dione derivatives from marine fungus Gliocladium sp. Pharmazie 2009, 64, 616-618.

39. Wang, Y.; Li, Z.L.; Bai, J.; Zhang, L.M.; Wu, X.; Zhang, L.; Pei, Y.H.; Jing, Y.K.; Hua, H.M. 2,5-Diketopiperazines from the marine-derived fungus Aspergillus fumigatus YK-7. Chem. Biodivers. 2012, 9, 385-393.

40. He, F.; Sun, Y.L.; Liu, K.S.; Zhang, X.Y.; Qian, P.Y.; Wang, Y.F.; Qi, S.H. Indole alkaloids from marine-derived fungus Aspergillus sydowii SCSIO 00305. J. Antibiot. 2012, 65, 109-111. 
41. Li, X.J.; Zhang, Q.; Zhang, A.L.; Gao, J.M. Metabolites from Aspergillus fumigatus, an endophytic fungus associated with melia azedarach, and their antifungal, antifeedant, and toxic activities. J. Agr. Food Chem. 2012, 60, 3424-3431.

42. Tsukamoto, S.; Umaoka, H.; Yoshikawa, K.; Ikeda, T.; Hirota, H. Notoamide O, a structurally unprecedented prenylated indole alkaloid, and notoamides $\mathrm{P}-\mathrm{R}$ from a marine-derived fungus, Aspergillus sp. J. Nat. Prod. 2010, 73, 1438-1440; Correction 2013, 76, 1232.

43. Kato, H.; Yoshida, T.; Tokue, T.; Nojiri, Y.; Hirota, H.; Ohta, T.; Williams, R.M.; Tsukamoto, S. Notoamides A-D: Prenylated indole alkaloids isolated from a marine-derived fungus, Aspergillus sp. Angew. Chem. Int. Edit. 2007, 46, 2254-2256.

44. Grubbs, A.W.; Artman, G.D.; Tsukamoto, S.; Williams, R.M. A concise total synthesis of the notoamides C and D. Angew. Chem. Int. Edit. 2007, 46, 2257-2261.

45. Tsukamoto, S.; Kato, H.; Greshock, T.J.; Hirota, H.; Ohta, T.; Williams, R.M. Isolation of notoamide E, a key precursor in the biosynthesis of prenylated indole alkaloids in a marine-derived fungus, Aspergillus sp. J. Am. Chem. Soc. 2009, 131, 3834-3835.

46. Tsukamoto, S.; Kawabata, T.; Kato, H.; Greshock, T.J.; Hirota, H.; Ohta, T.; Williams, R.M. Isolation of antipodal (-)-versicolamide $\mathrm{B}$ and notoamides $\mathrm{L}-\mathrm{N}$ from a marine-derived Aspergillus sp. Org. Lett. 2009, 11, 1297-1300.

47. Schultz, A.W.; Lewis, C.A.; Luzung, M.R.; Baran, P.S.; Moore, B.S. Functional characterization of the cyclomarin/cyclomarazine prenyltransferase CymD directs the biosynthesis of unnatural cyclic peptides. J. Nat. Prod. 2010, 73, 373-377.

48. Ding, Y.S.; de Wet, J.R.; Cavalcoli, J.; Li, S.Y.; Greshock, T.J.; Miller, K.A.; Finefield, J.M.; Sunderhaus, J.D.; McAfoos, T.J.; Tsukamoto, S.; et al. Genome-based characterization of two prenylation steps in the assembly of the stephacidin and notoamide anticancer agents in a marine-derived Aspergillus sp. J. Am. Chem. Soc. 2010, 132, 12733-12740.

49. McAfoos, T.J.; Li, S.Y.; Tsukamoto, S.; Sherman, D.H.; Williams, R.M. Studies on the biosynthesis of the stephacidins and notoamides. Total synthesis of notoamide S. Heterocycles 2010, 82, 461-472.

50. Afiyatullov, S.S.; Zhuravleva, O.I.; Chaikina, E.L.; Anisimov, M.M. A new spirotryprostatin from the marine isolate of the fungus Aspergillus fumigatus. Chem. Nat. Compd. 2012, 48, 95-98.

51. He, F.; Han, Z.; Peng, J.; Qian, P.Y.; Qi, S.H. Antifouling indole alkaloids from two marine derived fungi. Nat. Prod. Commun. 2013, 8, 329-332.

52. Tsukamoto, S.; Kato, H.; Samizo, M.; Nojiri, Y.; Onuki, H.; Hirota, H.; Ohta, T. Notoamides F-K, prenylated indole alkaloids isolated from a marine-derived Aspergillus sp. J. Nat. Prod. 2008, 71, 2064-2067.

53. Qian-Cutrone, J.F.; Huang, S.; Shu, Y.Z.; Vyas, D.; Fairchild, C.; Menendez, A.; Krampitz, K.; Dalterio, R.; Klohr, S.E.; Gao, Q. Stephacidin A and B: Two structurally novel, selective inhibitors of the testosterone-dependent prostate LNCaP cells. J. Am. Chem. Soc. 2002, 124, $14556-14557$.

54. Yang, B.; Dong, J.D.; Zhou, X.F.; Yang, X.W.; Lee, K.J.; Wang, L.S.; Zhang, S.; Liu, Y.H. Proline-containing dipeptides from a marine sponge of a Callyspongia species. Helv. Chim. Acta 2009, 92, 1112-1117. 
55. Ovenden, S.P.B.; Nielson, J.L.; Liptrot, C.H.; Willis, R.H.; Tapiolas, D.M.; Wright, A.D.; Motti, C.A. A new diketopiperazine, cyclo-(4-S-hydroxy- $R$-proline- $R$-isoleucine), from an Australian specimen of the sponge Stelletta sp. Mar. Drugs 2011, 9, 2469-2478.

56. Chen, Y.N.; Peng, Y.; Gao, C.H.; Huang, R.M. A new diketopiperazine from South China Sea marine sponge Callyspongia sp. Nat. Prod. Res. 2014, 28, 1010-1014.

57. Gao, C.H.; Lin, L.; Long, B.; Chen, Y.N.; He, B.J.; Sun, H.Y.; Huang, R.M. A new diketopiperazine from the gorgonian coral Menella kanisa. Nat. Prod. Res. 2014, 28, 473-476.

58. Xu, X.L.; Yin, L.Y.; Fang, N.Q.; Fan, X.; Song, F.H. Bromophenol coupled with diketopiperazine from marine red alga Symphyocladia latiuscula. Chem. Nat. Compd. 2012, 48, 622-624.

59. Blunt, J.W.; Copp, B.R.; Keyzers, R.A.; Munro, M.H.G.; Prinsep, M.R. Marine natural products. Nat. Prod. Rep. 2014, 31, 160-258.

60. Blunt, J.W.; Copp, B.R.; Keyzers, R.A.; Munro, M.H.G.; Prinsep, M.R. Marine natural products. Nat. Prod. Rep. 2013, 30, 237-323.

(C) 2014 by the authors; licensee MDPI, Basel, Switzerland. This article is an open access article distributed under the terms and conditions of the Creative Commons Attribution license (http://creativecommons.org/licenses/by/4.0/). 